\title{
JEAN-FRANÇOIS JAULENT
}

\section{La théorie de Kummer et le $K_{2}$ des corps de nombres}

Séminaire de Théorie des Nombres de Bordeaux, tome 2, nº 2 (1990), p. $377-411$

<http://www.numdam.org/item?id=JTNB_1990_2_2_377_0>

(C) Université Bordeaux 1, 1990, tous droits réservés.

L'accès aux archives de la revue "Séminaire de Théorie des Nombres de Bordeaux » (http://jtnb.cedram.org/) implique l'accord avec les conditions générales d'utilisation (http://www.numdam.org/conditions). Toute utilisation commerciale ou impression systématique est constitutive d'une infraction pénale. Toute copie ou impression de ce fichier doit contenir la présente mention de copyright.

\section{Numdam}

Article numérisé dans le cadre du programme

Numérisation de documents anciens mathématiques

http://www.numdam.org/ 
Séminaire de Théorie des Nombres, Bordeaux 2 (1990), 377-411

\title{
La théorie de Kummer et le $K_{2}$ des corps de nombres
}

\author{
par JEAN-FranÇOIS JAULENT
}

Résumé : Nous associons à chaque corps de nombres $K$ un groupe universel $\overline{K_{2}}(K)$ analogue au groupe symbolique $K_{2}(K)$, et deux sous-groupes canoniques finis $\overline{R_{2}}(K)$ et $\overline{H_{2}}(K)$, qui correspondent aux noyaux réguliers et hilbertien de la $K$-théorie, et permettent d'expliciter les correspondances remarquables entre divers modules galoisiens classiques faisant intervenir les conjectures de Leopoldt et de Gross.

\section{SOMMAIRE}

\section{INTRODUCTION}

\section{CONVENTIONS \& NOTATIONS}

\section{1. - LE RADICAL UNIVERSEL $\mathfrak{R}$.}

a.- Définition des groupes $\overline{K_{2}}\left(K_{n}\right)$.

b.- Introduction des groupes d'unités logarithmiques.

c.- Application aux groupes de Brauer.

d.- Restriction au radical modéré (ou régulier) $\mathfrak{M}$.

\section{2. - LE RADICAL HILBERTIEN $\mathfrak{H}$}

a.- Interprétation symbolique : les groupes $H_{2}\left(K_{n}\right)$.

b.- Interprétation kummérienne : les groupes $\overline{H_{2}}\left(K_{n}\right)$.

c.- Application aux unités logarithmiques.

d.- Isomorphismes de fausse dualité.

APPENDICE : LES CONJECTURES DE LEOPOLDT ET DE GROSS

INDEX DES NOTATIONS

RÉFÉRENCES

Manuscrit reçu le 12 mai 1990. 


\section{INTRODUCTION}

Nous développons dans cet article un parallèle entre la théorie de Tate sur le $K_{2}$ des corps de nombres et la description kummérienne des genres dans une tour cyclotomique. Nous sommes amenés, pour cela, à associer à chaque corps de nombres $K$ un groupe universel $\overline{K_{2}}(K)$, analogue au groupe symbolique $K_{2}(K)$, et deux sous-groupes canoniques finis, $\overline{R_{2}}(K)$ et $\overline{H_{2}}(K)$, qui correspondent respectivement aux noyaux réguliers $R_{2}(K)$ et hilbertien $\mathrm{H}_{2}(K)$ de la $K$-théorie. Ces deux groupes sont remarquables à plus d'un titre :

- D'abord, comme le montrent les résultats de fausse dualité énoncés dans la dernière section, ils constituent une bonne approximation des noyaux de la $K$-théorie : en particulier, ils ont les mêmes $\ell^{m}$-rangs dès que le corps $K$ contient les racines $\ell^{m}$-ièmes de l'unité.

- Ensuite, puisque définis kummériennement, ils relèvent par dualité des méthodes de la théorie du corps de classes, et se présentent en fin de compte comme les duaux de Pontrjagin de modules galoisiens classiques convenablement tordus, dont l'arithmétique est bien connue.

- Enfin, il se trouve qu'ils interviennent de façon naturelle dans un certain nombre de questions mettant en jeu les conjectures de Leopoldt et de Gross, pour lesquelles ils fournissent un cadre conceptuel particulièrement commode.

Une première rédaction de cette étude figure dans le chapitre I de [13]. La présente version, plus complète, intègre nombre de résultats récents, et met plus particulièrement l'accent sur le rôle joué dans les problèmes étudiés par le $\ell$-groupe des classes logarithmiques introduit dans [14] mais déjà rencontré dans [13] sous une forme légèrement différente (c'est le groupe des classes de valeurs absolues). Un court appendice présente dans ce contexte une condition suffisante classique des conjectures de Leopoldt et de Gross.

\section{CONVENTIONS \& NOTATIONS}

Dans tout ce qui suit, $\ell$ désigne un nombre premier (éventuellement 2), et $K$ un corps de nombres contenant les racines $2 \ell$-ièmes de l'unité.

Nous écrivons $K_{\infty}=\bigcup_{n \in \mathbb{N}} K_{n}$ la $\ell$-tour cyclotomique construite sur $K$, avec la convention d'indice $K_{n}=K\left[\mu_{\ell^{n}}\right]$, où $\mu_{\ell^{n}}$ désigne le groupe des 
racines $\ell^{n}$-ièmes de l'unité dans $\mathbb{C}$. Nous notons $\mu_{\ell^{\infty}}=\bigcup_{n \in \mathbb{N}} \mu_{\ell^{n}}$ la limite inductive des $\mu_{\ell^{n}}$, puis $\mathbf{T}_{\ell}=\longleftarrow \lim _{\ell^{n}}$ le module de Tate (qui s'identifie à $\mathbb{Z}_{\ell}$ comme groupe abstrait, via l'exponentielle complexe $\left.n \mapsto \exp \frac{2 i \pi}{n}\right)$, et $\overline{\mathbf{T}}_{\ell}$ le module opposé (c'est à dire le même groupe équipé de l'action opposée des groupes de Galois), qui s'identifie canoniquement au dual de Pontrjagin $\hat{\mu}_{\ell \infty}=\operatorname{Hom}_{\mathbf{Z}}\left(\mu_{\ell \infty}, \mathbf{Q}_{\ell} / \mathbf{Z}_{\ell}\right)$ du groupe $\mu_{\ell \infty}$.

Le caractère $\omega$ de l'action du groupe de Galois $\operatorname{Gal}\left(K_{\infty} / K\right)$ sur le mod-

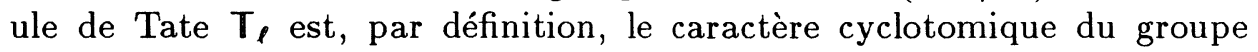
$\operatorname{Gal}\left(K_{\infty} / K\right)$; son opposé $\bar{\omega}$, défini par

$$
\bar{\omega}(\sigma)=\omega\left(\sigma^{-1}\right), \forall \sigma \in \operatorname{Gal}\left(K_{\infty} / K\right)
$$

est le caractère anticyclotomique ; c'est aussi le caractère du module $\overline{\mathbf{T}}_{\ell}$.

Si $n_{0}$ est le plus grand entier qui vérifie $K_{n}=K$ (de sorte que $K$ contient $\mu_{\ell^{n_{0}}}$ mais non $\mu_{\ell^{n_{0}+1}}$, nous supposerons implicitement dans tout ce qui suit $n \geq n_{0}$, et donc $\left[K_{n+1}: K_{n}\right]=\ell$. En particulier, par $\gamma_{n}$ nous entendons l'unique générateur topologique du groupe procyclique $\Gamma_{n}=\operatorname{Gal}\left(K_{\infty} / K_{n}\right)$ défini par l'identité

$$
\zeta^{\gamma_{n}}=\zeta^{1+\ell^{n}}, \text { pour tout } \zeta \text { de } \mu_{\rho \infty},
$$

c'est à dire par la condition $\omega\left(\gamma_{n}\right)=1+\ell^{n}$.

Convention : Pour tout module multiplicatif $\mathfrak{X}$, nous disons qu'un élément $x$ de $\mathfrak{X}$ est : i) de hauteur $m$, lorsque c'est une puissance $\ell^{m}$-ième dans $\mathfrak{X}$, i.e. lorsqu'il s'écrit $x=x_{m}^{\ell^{m}}$ pour un $x_{m}$ de $\mathfrak{X}$;

ii) de hauteur $\infty$, lorsqu'il est de hauteur $m$ pour tout $m$, i.e. lorsque pour tout $m \in \mathbb{N}$, il existe un $x_{m} \in \mathfrak{X}$ qui vérifie $x=x_{m}^{p^{m}}$;

iii) divisible, lorsqu'il peut être indéfiniment divisé, i.e. lorsqu'il existe une suite $\left(x_{m}\right)_{m \in \mathbb{N}} \in \mathfrak{X}^{\mathbb{N}}$, avec $x=x_{1}^{\ell}, x_{1}=x_{2}^{\ell}$, etc...

Nous notons $\mathfrak{X}_{\infty}=\bigcap_{m \in \mathbb{N}} \mathfrak{X}^{\ell^{m}}$ le sous-module de $\mathfrak{X}$ formé des éléments de hauteur infinie, et $\mathfrak{X}_{\text {div }}$ le sous-module de $\mathfrak{X}_{\infty}$ constituté des éléments divisibles de $\mathfrak{X}$.

Le module $\mathfrak{X}_{\text {div }}$ coïndide avec $\mathfrak{X}_{\infty}$, lorsque $\mathfrak{X}$ est de cotype fini (i.e. lorsque le sous-module $\mathfrak{X}$ annulé par $\ell$ est fini), mais ce résultat est évidemment faux en général.

Enfin, suivant l'usage, nous appelons codimension d'un module divisible de torsion $\mathfrak{X}$ la dimension sur $\mathbb{Z}_{\ell}$ de son dual de Pontrjagin $\hat{\mathfrak{X}}=$ $\operatorname{Hom}\left(\mathfrak{X}, \mathbf{Q}_{\ell} / \mathbf{Z}_{\ell}\right)$. Lorsque $\mathfrak{X}$ est de cotype fini et stable pour l'action d'un groupe d'automorphismes $\Delta$, nous disons de même que le caractère du $\mathbb{Q}_{\ell}[\Delta]$-module $\mathbb{Q}_{\ell} \otimes_{\mathbb{Z}}, \hat{\mathfrak{X}}$ est le caractère défini par $\mathfrak{X}$. 


\section{1. - LE RADICAL UNIVERSEL $\mathfrak{R}$}

Définition 1.1. Par $\ell$-radical universel d'un corps de nombres $K$, nous entendons le tensorisé par $\mathbb{Q}_{\ell} / \mathbb{Z}_{\ell}$ de son groupe multiplicatif, que nous notons :

$$
\mathfrak{R}_{K}=\left(\mathbb{Q}_{\ell} / \mathbb{Z}_{\ell}\right) \otimes_{\mathbb{Z}} K^{\times}
$$

Le groupe $\mathfrak{R}_{K^{-}}$est, par construction, un $\mathbb{Z}_{\ell}$-module divisible de torsion, dont tout élément admet une représentation de la forme $\ell^{-m} \otimes x$ avec $m \in \mathbb{N}$ et $x \in K^{\times}$. La condition $\ell^{-m} \otimes x=1$ signifie alors que l'élément $x$ est le produit $x=\zeta y^{\ell^{m}}$ d'une racine de l'unité et d'une puissance $\ell^{m}$ ième dans $K^{\times}$. On prendra garde, en effet, que le produit tensoriel par le groupe divisible $\mathbb{Q}_{\ell} / \mathbb{Z}_{\ell}$ a pour conséquence la disparition du sous-groupe de torsion de $K^{\times}$. Dans le cas qui nous intéresse, la tour cyclotomique $K_{\infty}$ étant la réunion des sous-corps $K_{n}$, le radical universel, disons $\mathfrak{R}_{K_{\infty}}$, qui lui correspond s'obtient naturellement comme limite inductive des radicaux $\mathfrak{R}_{K_{n}}=\left(\mathbb{Q}_{\ell} / \mathbb{Z}_{\ell}\right) \otimes_{\mathbb{Z}} K_{n}^{\times}$. Le point essentiel ici est que les groupes $\mathfrak{R}_{K_{n}}$ vérifient la théorie de Galois :

Proposition 1.2. L'application naturelle de $\mathfrak{R}_{K_{n}}$ dans $\Re_{K_{\infty}}$ est un morphisme injectif. Plus précisément, pour chaque naturel $n$, le radical universel $\mathfrak{R}_{K_{n}}$ associé au corps $K_{n}$ s'identifie canoniquement au sous-module des points fixes dans $\mathfrak{R}_{K_{\infty}} d u$ groupe de Galois $\Gamma_{n}=\operatorname{Gal}\left(K_{\infty} / K_{n}\right)$; ce que nous écrivons tout simplement :

$$
\mathfrak{R}_{\kappa_{n}}=\mathfrak{R}_{\kappa_{\infty}}^{\Gamma_{n}}, \quad \forall n \in \mathbb{N} .
$$

Démonstration (cf. [7], section II, pour un cas particulier) : Soit $x_{n}$ un élément de $K_{n}^{\times}$. La relation $\ell^{-1} \otimes x_{n}=1$ dans $\Re_{K_{\infty}}$ entraîne $K_{n}\left[{ }^{\ell} \sqrt{x} \subset\right.$ $K_{\infty}\left[{ }^{\ell} \sqrt{x}\right]=K_{\infty}$, donc, par la théorie de Kummer : $x_{n} \equiv \zeta_{n}\left(\bmod ^{\times} K_{n}^{\times \ell}\right)$, pour un $\zeta_{n}$ de $\mu_{\ell^{n}}$; c'est à dire, en fin de compte, $\ell^{-1} \otimes x_{n}=1$ dans $\Re_{K_{n}}$. Autrement dit, tout élément d'ordre $\ell$ de $\mathfrak{R}_{K_{n}}$ s'envoie sur un élément d'ordre $\ell$ de $\mathfrak{R}_{K_{\infty}}$, et l'application naturelle de $\mathfrak{R}_{K_{n}}$ dans $\mathfrak{R}_{K_{\infty}^{-}}$est bien injective.

Soit maintenant $\ell^{-p} \otimes x_{p}$ un élément de $\Re_{K_{p}}$ fixé par $\Gamma_{n}$, pour un $n \leq p$. Dans ce cas, $\ell^{-p} \otimes x_{p}^{\gamma_{n}-1}$ est égal à 1 , ce qui signifie (puisque les racines de l'unité sont divisibles dans $K_{\infty}^{\times}$) que $x_{p}^{\gamma_{n}-1}$ est une puissance $\ell^{p}$-ième dans $K_{\infty}^{\times}$. Écrivons donc $x_{p}^{\gamma_{n}-1}=y_{q}^{\ell^{n}}$ pour un $y_{q}$ de $K_{q}^{\times}$, avec $q \geq n$; et considérons l'élément $N_{q / n}\left(y_{q}\right)$ donné par la norme arithmétique $N_{q / n}=$ $N_{K_{q} / K_{n}}$. Nous avons $N_{q / n}\left(y_{q}\right)^{\ell^{p}}=N_{q / n}\left(x_{p}^{\gamma_{n}-1}\right)=x_{p}^{\gamma_{q}-1}=1$, ce qui prouve que $N_{q / n}\left(y_{q}\right)$ est une racine de l'unité dans $K_{n}^{\times}$, et, par conséquent la 
norme $N_{q / n}\left(\zeta_{q}\right)$ d'une racine de l'unité $\zeta_{q}$ de $K_{q}^{\times}$. Il suit : $N_{q / n}\left(y_{q} / \zeta_{q}\right)=1$, puis, en vertu du théorème 90 de Hilbert appliqué à l'extension cyclique $K_{q} / K_{n}, y_{q}=\zeta_{q} z_{q}^{\gamma_{n}-1}$, pour un $z_{q}$ de $K_{q}^{\times}$. En particulier, l'élément

$\left(x_{p} z_{q}^{-\ell^{p}}\right)^{\gamma_{n}-1}=\zeta_{q}^{\ell^{p}}$ est une racine de l'unité, de sorte que nous pouvons écrire $\left(x_{p} z_{q}^{-\rho^{p}}\right)^{\gamma_{n}-1}=\zeta_{q}^{\rho^{p-n}\left(\gamma_{n}-1\right)}$ ce qui nous prouve que $x_{p}$ est le produit $x_{n} \zeta_{q}^{\ell^{p-n}} z_{q}^{\ell^{p}}$ d'un élément $x_{n}$ de $K_{n}^{\times}$, d'une racine de l'unité, et d'une puissance $\ell^{p}$-ième ; autrement dit que nous avons $\ell^{-p} \otimes x_{p}=\ell^{-p} \otimes x_{n} \in \mathfrak{R}_{K_{n}}$, comme attendu.

Corollaire 1.3. Lorsque $\ell$ est impair, l'intersection des sous-modules respectifs de $\mathbf{T}_{\ell} \otimes_{\mathbb{Z}}, \mathfrak{R}_{K_{\infty}}$ et $\overline{\mathbf{T}}_{\boldsymbol{\ell}} \otimes_{\mathbb{Z}}, \mathfrak{R}_{K_{\infty}^{\prime}}$ fixés par $\Gamma_{n}$ est le sous-groupe de $\ell^{n}$-torsion de $\mathfrak{R}_{K_{n}}$ :

$$
\left(\mathbb{T}_{\ell} \otimes \mathbb{Z}, \mathfrak{R}_{K_{\infty}}\right)^{\Gamma_{n}} \cap\left(\bar{T}_{\ell} \otimes_{\mathbb{Z}}, \mathfrak{R}_{K_{\infty}}\right)^{\Gamma_{n}}=\ell^{n} \mathfrak{R}_{K_{\infty}^{n}}^{\Gamma_{n}}=\ell^{n} \mathfrak{R}_{K_{n}} .
$$

Pour $\ell=2$, en revanche, on a seulement

$$
\left(\mathbf{T}_{2} \otimes \mathbb{Z}_{2} \mathfrak{R}_{K_{\infty}}\right)^{\Gamma_{n}} \cap \mathfrak{R}_{\kappa_{\infty}^{n}}^{\Gamma_{n}}={ }_{2^{n}} \mathfrak{R}_{K_{n}}=\left(\overline{\mathbf{T}}_{2} \otimes \mathbb{Z}_{2} \mathfrak{R}_{K_{\infty}}\right)^{\Gamma_{n}} \cap \mathfrak{R}_{K_{\infty} \Gamma_{\infty}} .
$$

Démonstration : Les modules $\mathbb{Z}_{\ell}, \mathbf{T}_{\ell}$, et $\overline{\mathbf{T}}_{\ell}$ étant identiques en tant que groupes abstraits, les groupes $\mathfrak{R}_{K_{\infty}^{\prime}}, \mathbf{T}_{\boldsymbol{\ell}} \otimes_{\mathbb{Z}_{\ell}} \mathfrak{R}_{K_{\infty}^{\prime}}$, et $\overline{\mathbf{T}}_{\boldsymbol{l}} \otimes_{\mathbb{Z}}, \mathfrak{R}_{K_{\infty}}$ ne diffèrent que par l'action de Galois. Soit donc $\ell^{-p} \otimes x_{p}$ un élément de l'intersection $\left(\mathbb{T}_{\boldsymbol{\ell}} \otimes_{\mathbb{Z}}, \mathfrak{R}_{K_{\infty}}\right)^{\Gamma_{n}} \cap\left(\overline{\mathbf{T}}_{\boldsymbol{\ell}} \otimes_{\mathbb{Z}}, \mathfrak{R}_{K_{\infty}}\right)^{\Gamma_{n}}$. L'identité $\ell^{-\boldsymbol{p}} \otimes$ $x_{p}^{\gamma_{n}-\omega\left(\gamma_{n}\right)}=1=\ell^{-p} \otimes x_{p}^{\gamma_{n}^{-1}-\omega\left(\gamma_{n}\right)}$ nous montre que $\ell^{-p} \otimes x_{p}$, qui est annulé par $\gamma_{n}-\gamma_{n}^{-1}$, est fixé par $\gamma_{n}^{2}$, donc contenu dans $\mathfrak{R}_{K_{n}}$ d'après la proposition 1 ci-dessous dès lors que $\ell$ est impair. Nous pouvons ainsi supposer $x_{p} \in K_{n}^{\times}$, auquel cas nous obtenons immédiatement :

$$
\ell^{-p+n} \otimes x_{p}=\ell^{-p} \otimes x_{p}^{\ell^{n}}=\ell^{-p} \otimes x_{p}^{\omega \cdot\left(\gamma_{n}\right)-\gamma_{n}}=1,
$$

i.e. $\ell^{-p} \otimes x_{p} \in_{\ell^{n}} \mathfrak{R}_{\kappa_{n}}$ ce qui achève la démonstration dans ce cas. Le cas $\ell=2$ est immédiat.

\section{a. - Définition des groupes $\overline{K_{2}}\left(K_{n}\right)$}

Le groupe universel $K_{2}(K)$ pour les symboles sur un corps $K$ peut être défini comme le quotient du carré tensoriel $K^{\times} \otimes_{\mathbb{Z}} K^{\times}$du $\mathbb{Z}$-module multiplicatif $K^{\times}$par l'idéal engendré par les éléments de la forme $x \otimes(1-x)$, pour $x \in K \backslash\{0,1\}$. Dans le cas des corps de nombres, on sait depuis les 
résultats de Tate et Bass, et le théorème de finitude de Garland (cf.[2]) que $K_{2}(K)$ est un groupe de torsion, donc le produit direct de ses sous-groupes de Sylow. En fait, seul le $\ell$-sous-groupe de Sylow nous importe ici, de sorte que nous continuerons par abus à le noter $K_{2}(K)$ pour ne pas alourdir inutilement les notations. Cela posé, le résultat fondamental de Tate (cf.[24] et [26]) est alors que les éléments d'ordre $\ell$-primaire de $K_{2}(K)$ (c'est-à-dire ceux de $K_{2}(K)$, avec la convention d'écriture ci-dessus) s'obtiennent par montée dans la $\ell$-tour cyclotomique $K_{\infty}$ :

Théorème 1.4. Pour chaque naturel $n$, le $\ell$-sous-groupe de Sylow $K_{2}\left(K_{n}\right)$ du groupe universel pour les symboles sur le corps $K_{n}$ est déterminé par la

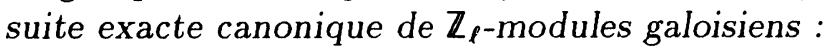

$$
1 \longrightarrow\left(\mathrm{T}_{\ell} \otimes \mathbb{Z}, \mathfrak{R}_{K_{\infty}}\right)_{\mathrm{div}}^{\Gamma_{n}} \longrightarrow\left(\mathrm{T}_{\ell} \otimes \mathbb{Z}_{\ell} \mathfrak{R}_{K_{\infty}}\right)^{\Gamma_{n}} \longrightarrow K_{2}\left(K_{n}\right) \longrightarrow 1
$$

Dans celle-ci, le terme médian désigne le sous-groupe des points fixes par $\Gamma_{n}=\operatorname{Gal}\left(K_{\infty} / K_{n}\right)$ du produit tensoriel $\mathbf{T}_{\ell} \otimes_{\mathbb{Z}}, \mathfrak{R}_{K_{\infty}} \simeq \mu_{\rho \infty} \otimes_{\mathbb{Z}} K_{\infty}^{\times}$, et le terme de gauche son sous-groupe divisible maximal.

Le calcul de la codimension du sous-groupe divisible $\left(T_{\ell} \otimes_{\mathbb{Z}}, \mathfrak{R}_{K_{\infty}}\right)_{\text {div }}^{\Gamma_{n}}$ est l'un des théorèmes essentiels de Tate sur le $K_{2}$ des corps de nombres (cf. [26], §5). Comme nous allons le voir plus loin, ce résultat est l'analogue pour la $K$-théorie des corps de nombres, de la conjecture de Leopoldt pour la $\ell$-ramification. Avant de développer ce point de vue, disons brièvement comment le résultat de Tate peut s'énoncer de façon sensiblement plus précise en termes de représentations : Donnons nous un groupe (fini), disons $\Delta$, d'automorphismes de $K$, et notons $F$ son corps des invariants : puis pour chaque entier naturel $n$, écrivons $\Delta_{n} \simeq \Delta$ le relèvement naturel de $\Delta$ dans $K_{n}$, et $F_{n}$ son sous corps des points fixes. Pour chaque place à l'infini $\mathfrak{p}_{n}$ du corps $F_{n}$, désignons par $\Delta_{\mathfrak{p}_{n}}$ un sous-groupe de décomposition dans l'extension $K_{n} / F_{n}$, lequel ne dépend en fait que de la place $\mathfrak{p}_{0}$ de $F_{o}=F$ au dessous de $\mathfrak{p}_{n} ;$ notons $\chi_{\mathfrak{p}_{n}}=\operatorname{Ind} d_{\Delta_{\mathfrak{p}_{n}}}^{\Delta} 1_{\Delta_{\mathfrak{p}_{n}}}$ l'induit à $\Delta$ du caractère de la représentation unité de $\Delta_{p_{n}} \simeq \Delta_{p_{n}}$; et formons la somme

$$
\sum_{\mathfrak{p}_{n} \mid \infty} \chi_{\mathfrak{p}_{n}}=\left[K_{n}: K_{o}\right] \sum_{\mathfrak{p}_{o} \mid \infty} \chi_{\mathfrak{p}_{n}} .
$$

D'après les résultats de Tate (cf. [26]), le caractère obtenu est celui du noyau universel $\mathfrak{U}_{K_{n}}$ défini par l'identité :

$$
\mathrm{T}_{\ell} \otimes \mathbb{Z}, \mathfrak{U}_{\kappa_{n}^{-}}={ }_{\text {déf }}\left(\mathrm{T}_{\ell} \otimes \mathbb{Z}, \mathfrak{R}_{K_{\infty}}\right)_{\text {div }}^{\Gamma_{n}}
$$


Scolie 1.5. Le sous-groupe divisible maximal $\left(T_{\ell} \otimes_{\mathbb{Z}}, \mathfrak{R}_{K_{\infty}^{-}}\right)_{\mathrm{div}}^{\Gamma_{n}}$ du groupe $\left(T_{\ell} \otimes \mathbb{Z}_{,} \Re_{K_{\infty}}\right)^{\Gamma_{n}}$ est un $\mathbb{Z}_{\ell}$-module divisible de codimension finie, égale au nombre de places complexes $c_{K_{n}}$ du corps $K_{n}$ :

$$
\left(T_{\ell} \otimes \mathbb{Z}, \mathfrak{R}_{K_{\infty}}\right)_{\text {div }}^{\Gamma_{n}} \simeq\left(\mathbb{Q}_{\ell} / \mathbb{Z}_{\ell}\right)^{c K_{n}}
$$

Plus précisément, pour tout groupe (fini) $\Delta_{n}$ d'automorphismes de $K_{n}$, le caractère de $\Delta_{n}$ associé au $\mathbb{Z}_{\ell}$-module divisible $\mathfrak{U}_{\kappa_{n}}$ défini par l'identité

$$
, \mathrm{T}_{\ell} \otimes \mathbf{Z}, \mathfrak{U}_{K_{n}}=\left(\mathrm{T}, \otimes \mathfrak{R}_{K_{\infty}}\right)_{d i v}^{\Gamma_{n}}
$$

est la somme $\sum_{\mathfrak{p}_{n} \mid \infty} \chi_{\mathfrak{p}_{n}}$ des induits à $\Delta_{n}$ des caractères unités des sous groupes de décomposition des places à l'infini dans l'extension relative attachée à $\Delta_{n}$.

THÉORÈME ET DÉFINITION 1.6. Par $\overline{K_{2}}\left(K_{n}\right)$ nous entendons le quotient du groupe $\left(\overline{\mathbf{T}}_{\ell} \otimes \mathbf{Z}, \mathfrak{R}_{K_{\infty}}\right)^{\Gamma_{n}}=\left(\bar{\mu}_{\ell \infty} \otimes_{\mathbb{Z}} K_{\infty}^{\times}\right)^{\Gamma_{n}}$ par son sous-module divisible maximal, c'est-à-dire le $\ell$-groupe de torsion défini par l'exactitude de la suite courte :

$$
1 \longrightarrow\left(\overline{\mathbf{T}}, \otimes_{\mathbf{Z}}, \mathfrak{R}_{K_{\infty}}\right)_{\mathrm{div}}^{\Gamma_{n}} \longrightarrow\left(\overline{\mathbf{T}}, \otimes_{\mathbb{Z}}, \mathfrak{R}_{K_{\infty}}\right)^{\Gamma_{n}} \longrightarrow \overline{K_{2}}\left(K_{n}\right) \longrightarrow 1 .
$$

Le groupe $\overline{K_{2}}\left(K_{n}\right)$ ainsi obtenu décrit, via la théorie de Kummer, l'ensemble des $\ell$-extensions de $K_{\infty}$ qui proviennent par composition avec $K_{\infty}$ d'une extension abélienne de $K_{n}$ modulo celles qui proviennent des $\mathbb{Z}_{\ell}$-extensions de $K_{n}$.

Démonstration : Désignons par $\mathcal{X}_{K_{\infty}}$ le groupe de Galois de la $\ell$-extension abélienne maximale de $K_{\infty}$, puis par $\Gamma^{\Gamma_{n}} \mathcal{X}_{K_{\infty}}$ son quotient des genres relatif à l'extension procyclique $K_{\infty} / K$, i.e le plus grand quotient de $\mathcal{X}_{\infty}$ sur lequel $\Gamma_{n}$ opère trivialement, autrement dit $\mathcal{X}_{\kappa_{\infty}} / \mathcal{X}_{K_{\infty}}^{\gamma_{n}-1}:$ c'est aussi le groupe de Galois $\operatorname{Gal}\left(K_{n}^{a b} / K_{\infty}\right)$ attaché à la plus grande $\ell$-extension $K_{n}^{a b}$ de $K_{\infty}$ qui est abélienne sur $K_{n}$. Cela posé, nous avons immédiatement :

$$
\begin{aligned}
\left(\overline{\mathbf{T}}, \otimes_{\mathbb{Z}}, \mathfrak{R}_{K_{\infty}}\right)^{\Gamma_{n}} & =\left(\overline{\mathbf{T}}_{\ell} \otimes_{\mathbb{Z}}, \operatorname{Hom}\left(\mathcal{X}_{\kappa_{\infty}}, \mu_{\ell \infty}\right)\right)^{\Gamma_{n}} \\
& =\operatorname{Hom}_{\Gamma_{n}}\left(\mathcal{X}_{\kappa_{\infty}}, \mathbb{Q}_{\ell} / \mathbb{Z}_{\ell}\right)=\operatorname{Hom}\left(\Gamma_{n}, \mathcal{X}_{\kappa_{\infty}}, \mathbb{Q}_{\ell} / \mathbb{Z}_{\ell}\right) .
\end{aligned}
$$

Et le groupe $\left(\overline{\mathbf{T}}_{\ell} \otimes \boldsymbol{z}, \mathfrak{R}_{K_{\infty}}\right)^{\Gamma_{n}}$ est donc le dual de Pontrjagin de $\Gamma_{n} \mathcal{X}_{K_{\infty}}$, c'est-à-dire le tensorisé $\overline{\mathbf{T}}_{\ell} \otimes_{\mathbb{z}}, \operatorname{Rad}\left(K_{n}^{a h} / K_{\infty}\right)$ du radical kummérien annoncé ; son sous-groupe divisible maximal $\left(\overline{\mathbf{T}}, \otimes_{\mathbb{Z}}, \mathfrak{R}_{\kappa_{\infty}^{-}}\right)_{\text {div }}^{\Gamma_{n}}$ correspond, 
lui, au radical sur $K_{\infty}$ de la composée $Z_{n}$ des $\mathbb{Z}_{\ell}$-extensions de $K_{n}$. Il vient donc finalement :

$$
\overline{K_{2}}\left(K_{n}\right) \simeq \overline{\mathrm{T}}_{\ell} \otimes_{\mathbb{Z}}, \operatorname{Rad}\left(K_{n}^{a \cdot h} / Z_{n}\right)
$$

ce qui est précisément le résultat annoncé.

La conjecture de Leopoldt pour le corps $K_{n}$ (et le nombre premier $\ell$ ) affirme l'existence d'exactement $\left(c_{K_{n}}+1\right) \mathbb{Z}_{\ell}$-extensions linéairement indépendantes sur $K_{n}$, autrement dit l'égalité entre la codimension du groupe divisible $\left(\overline{\mathbf{T}}_{\ell} \otimes \mathbb{Z}, \mathfrak{R}_{K_{\infty}}\right)_{\mathrm{div}}^{\Gamma_{n}}$, et celle $c_{K_{n}}$ du groupe $\left(\boldsymbol{T}_{\ell} \otimes \mathbb{Z}_{\ell}, \mathfrak{R}_{K_{\infty}}\right)^{\Gamma_{n}}$ (donnée par le scolie 5$)$. On sait, de façon générale, que la codimension du radical $\left(\overline{\mathbf{T}}_{\ell} \otimes \mathbb{Z}, \Re_{K_{\infty}}\right)_{\infty}^{\Gamma_{n}}=\operatorname{Rad}\left(Z_{n} / K_{\infty}\right)$ n'éxède de $c_{K_{n}}$ que d'une quantité finie $\delta_{K_{n}}$, qui mesure donc le défaut de la conjecture de Leopoldt pour $K_{n}$, et qui reste d'ailleurs bornée lorsqu'on monte la tour cyclotomique $K_{\infty} / K_{0}$ (cf. [7], §1). Comme précédemment, ces résultats s'expriment en outre de façon plus précise en termes de représentations, la quantité $\delta_{K_{n}}$ s'interprétant comme degré du caractère de défaut pour la conjecture de Leopoldt dans le corps $K_{n}$ (cf. [13] IV.2, $3 \S \mathrm{b}$ ), le point remarquable étant que, sous la condition $\delta_{K_{n}}=0$ les groupes $\left(\overline{\mathbb{T}}_{\ell} \otimes \mathbb{Z}_{\ell} \mathfrak{R}_{K_{\infty}^{-}}\right)_{\mathrm{div}}^{\Gamma_{n}}$ et $\left(\mathbb{T}_{\ell} \otimes \mathbb{Z}_{1} \mathfrak{R}_{\kappa_{\infty}^{-}}\right)_{\mathrm{div}}^{\Gamma_{n}}$ définissent le même caractère.

SCOLIE 1.7. Le sous-groupe divisible maximal $\left(\overline{\mathbb{T}}_{\ell} \otimes_{\mathbb{Z}}, \mathfrak{R}_{\mathcal{K}_{\infty}^{-}}\right)_{\text {div }}^{\Gamma_{n}}$ du radical $\left(\overline{\mathbf{T}}_{\ell} \otimes \mathbb{Z}_{,} \mathfrak{R}_{K_{\infty}}\right)^{\Gamma_{n}}$ est un module de torsion de codimension finie égale au nombre de places complexes $c_{\kappa_{n}}$ du corps $K_{n}$ augmenté du défaut $\delta_{K_{n}}$ de la conjecture de Leopoldt dans $K_{n}$ :

$$
\left(\overline{\mathrm{T}}_{\ell} \otimes_{\mathbb{Z}}, \Re_{K_{\infty}}\right)_{\mathrm{div}}^{\Gamma_{n}} \simeq\left(\mathbb{Q}_{\ell} / \mathbb{Z}_{\ell}\right)^{c K_{n}}+\delta_{K^{\prime} n}
$$

Plus précisément, lorsque celle-ci est vérifiée, le noyau $\mathfrak{U}_{\kappa_{n}}$ des symboles universels et le radical $\mathfrak{Z}_{k_{n}}$ des $\mathbb{Z}_{\ell}$-extensions définis respectivement par

$$
\mathbb{T}_{\ell} \otimes \mathbb{Z}_{\ell} \mathfrak{U}_{K_{n}}=\left(\mathbb{T}_{\ell} \otimes_{\mathbb{Z}}, \mathfrak{R}_{\kappa_{\infty}}\right)_{\text {div }}^{\Gamma_{n}} \text { et } \bar{T}_{\ell} \otimes_{\mathbb{Z}} \mathfrak{Z}_{\kappa_{n}}=\left(\bar{T}_{\ell} \otimes_{\mathbb{Z}}, \mathfrak{R}_{\kappa_{\infty}}\right)_{\text {div }}^{\Gamma_{n}}
$$

ont même caractère, pour tout groupe (fini) $\Delta$ d'automorphismes de $K_{n}$.

\section{b. - Introduction des groupes d'unités logarithmiques}

Le nombre premier $\ell$ étant toujours supposé fixé, rappelons qu'à chaque place non complexe $\mathfrak{p}$ d'un corps de nombres $K$ est attachée une valeur absolue $\ell$-adique (i.e. à valeurs dans le groupe des unités $\mathbb{Z}_{\ell}^{\times}$de l'anneau 
$\mathbb{Z}_{\ell}$ ) définie sur le sous groupe multiplicatif $K_{\mathrm{p}}^{\times}$du complété p-adique de $K$ par la formule :

$$
|x|_{\mathfrak{p}}=\left\{\begin{array}{l}
s g_{\mathfrak{p}}(x), \text { si } \mathfrak{p} \text { est réelle; } \\
N \mathfrak{p}^{-v_{\mathfrak{p}}(x)}, \text { si } \mathfrak{p} \text { est finie et étrangère à } \ell ; \\
N_{K_{\mathfrak{p}} / \mathbb{Q}_{\ell}}(x) N \mathfrak{p}^{-v_{\mathfrak{p}}(x)}, \text { si } p \text { divise } \ell ;
\end{array}\right.
$$

$\left(s g_{\mathfrak{p}}\right.$ étant la fonction signe attachée à la place réelle $\mathfrak{p}, v_{\mathfrak{p}}$ la valuation associée à la place modérée $\mathfrak{p}$, et $N \mathfrak{p}$ la norme absolue de l'idéal $\mathfrak{p}$ ) : c'est la valuation $\ell$-adique de Tate (cf. [27], ch. VI, $\$ 1$ ). Par composition avec la projection naturelle $\langle$.$\rangle de \mathbb{Z}_{\ell}^{\times}$sur son sous-groupe principal $1+2 \ell \mathbb{Z}_{\ell}$, on obtient la valeur absolue $\ell$-adique principale définie dans [13] (cf. I.1, 1§c), qui se prolonge, elle, au complété profini :

$$
\mathcal{K}_{\mathrm{p}}^{\times}=\underset{m}{\lim } K_{\mathrm{p}}^{\times} / K_{\mathrm{p}}^{\times \ell^{m}}
$$

Dans les deux cas, par composition avec le logarithme $\ell$-adique $L o g$ de $\mathbb{Z}_{\ell}^{\times}$dans $\mathbb{Z}_{\ell}$, ces deux valeurs absolues nous donnent un même morphisme $h_{\mathfrak{p}}=\log ||_{\mathfrak{p}}$ qui se factorise par le complété profini $\mathcal{K}_{\mathfrak{p}}^{\times}$et prend ses valeurs dans le groupe additif $\mathbb{Z}_{\ell}$.

DÉFInITION 1.8. Nous appelons groupe des diviseurs logarithmiques d'un corps de nombres $K$, et nous notons $\mathcal{D} \ell_{K^{k}}$, l'image canonique $d u \ell$-adifié

$$
\mathcal{J}_{K}=\prod_{\mathfrak{p}}^{r e s} \mathcal{K}_{\mathfrak{p}}^{\times}
$$

du groupe des idèles de $K$ (défini comme produit restreint des complétés profinis $K_{\mathfrak{p}}^{\times}$) par la famille des applications $h_{\mathfrak{p}}=\log ||_{\mathfrak{p}}$ lorsque $\mathfrak{p}$ parcourt l'ensemble des places finies de K. Par convention, le degré degd d'un diviseur logarithmique est la somme dans $\mathbb{Z}_{\ell}$ de ses composantes.

D'après [13] (cf. prop. 1.1.8), le sous-groupe $\mathcal{P} \ell_{K}$ de $\mathcal{D} \ell_{K^{\prime}}$, image par $h=\left(h_{\mathfrak{p}}\right)_{\mathfrak{p}}$ du sous-groupe principal $\mathcal{R}_{K^{-}}=\mathbb{Z}_{\ell} \otimes_{\mathbb{Z}} K^{\times}$de $\mathcal{J}_{K^{-}}$est formé de diviseurs de degré nul. Plus précisément :

SColie 1.9. Le sous-groupe principal $\mathcal{P} \ell_{K}$ de $\mathcal{D} \ell_{K}$ est contenu dans le sous-groupe $\widetilde{\mathcal{D} \ell}$ des diviseurs logarithmiques de degré 0. Le quotient correspondant

$$
{\widetilde{\mathcal{C}} \ell_{K}}={\widetilde{\mathcal{D}} \ell_{K}} / \mathcal{P} \ell_{K}
$$


est, par définition, le groupe des classes logarithmiques de $K$. La conjecture de Gross généralisée (pour le corps $K$ et le premier $\ell$ ) affirme que c'est un $\ell$-groupe fini.

Cela posé, introduisons le tensorisé $\ell$-adique $\mathcal{D}_{K}=\mathbb{Z}_{\ell} \otimes_{\mathbb{Z}} D_{K}$ du groupe des diviseurs de $K$. L'application naturelle div qui a un idèle principal $x \in \mathcal{R}_{K}$ associe le diviseur principal $\operatorname{div}(x)=\prod_{\mathfrak{p}} \mathfrak{p}^{v_{\mathfrak{p}}(x)}$ conduit à la suite exacte à quatre termes

$$
1 \longrightarrow \mathcal{E}_{K} \longrightarrow \mathcal{R}_{K} \stackrel{\operatorname{div}}{\longrightarrow} \mathcal{D}_{K} \longrightarrow \mathcal{C} \ell_{K} \longrightarrow 1,
$$

où le noyau à gauche est le tensorisé $\ell$-adique $\mathcal{E}_{K^{-}}=\mathbb{Z}_{\ell} \otimes_{\mathbb{Z}} E_{K}$ du groupe des unités de $K$, et le conoyau à droite n'est rien d'autre que le $\ell$-groupe $C \ell_{K}$ des classes de diviseurs de $K$ (i.e. le $\ell$-sous-groupe de $S y l o w$ du groupe des classes de diviseurs). La suite exacte analogue attachée à $h$

$$
1 \longrightarrow \mathcal{N}_{K} \longrightarrow \mathcal{R}_{K} \stackrel{h}{\rightarrow} \widetilde{\mathcal{D} \ell_{K}} \rightarrow \widetilde{\mathcal{C}}_{K} \longrightarrow 1
$$

définit un sous-module pur $\mathcal{N}_{K^{-}}$de $\mathcal{R}_{K^{-}}$, qu'il est commode d'appeler groupe des unités logarithmiques du corps $K$. Pour déterminer son rang, remarquons d'abord que l'expression de $h_{\mathrm{p}}$ aux places modérées (i.e. étrangères à $\ell)$ montre que $\mathcal{N}_{K^{-}}$, tout comme $\mathcal{E}_{K^{-}}$, est contenu dans le tensorisé $\ell$-adique $\mathcal{E}_{K^{-}}^{\prime}=\mathbb{Z}_{\ell} \otimes_{\mathbb{Z}} E_{K^{k}}^{\prime}$ du groupe des $\ell$-unités de $K$. Par restriction à $\mathcal{E}_{K}^{\prime}$, nous obtenons alors la suite exacte :

$$
1 \longrightarrow \mathcal{N}_{K} \longrightarrow \mathcal{E}_{K^{-}}^{\prime} \longrightarrow \tilde{\oplus}_{\mathfrak{l} \ell \ell} \log \left|\mathcal{K}_{\mathfrak{l}}^{\times}\right|_{\mathfrak{r}} \longrightarrow{\widetilde{\mathcal{C}} \ell_{K}}^{\longrightarrow} \mathcal{C} \ell_{K}^{\prime}
$$

(où $\widetilde{\oplus}_{\mathfrak{l} \ell \ell} \log \left|\mathcal{K}_{\mathfrak{l}}^{\times}\right|_{\mathfrak{l}}$ désigne le sous-groupe de $\widetilde{\mathcal{D} \ell_{K}}$ constitué des diviseurs logarithmiques de degré nul construits sur les places au-dessus de $\ell$, et $C \ell_{K}^{\prime}$ le $\ell$-groupe des $\ell$-classes de diviseurs de $K$, i.e. le quotient de $C \ell_{K}$ par le sous-groupe des diviseurs construits sur les places au dessus de $\ell$ ), qu'il convient de rapprocher de la suite classique

$$
1 \longrightarrow \mathcal{E}_{K^{-}} \longrightarrow \mathcal{E}_{K^{-}}^{\prime} \longrightarrow \oplus \mathfrak{l} \ell^{\mathbb{Z}_{\ell}} \longrightarrow \mathcal{C} \ell_{K^{-}} \longrightarrow \mathcal{C} \ell_{K^{-}}^{\prime} \longrightarrow 1
$$

Maintenant, le groupe $\widetilde{\mathcal{C}} \ell_{\kappa}$ est fini sous la conjecture de Gross, et le terme médian $\widetilde{\oplus}_{\mathfrak{l} \mid \ell} \log \left|\mathcal{K}_{\mathfrak{l}}^{\times}\right|_{\mathfrak{r}}$ s'identifie (non canoniquement) au noyau de la for-

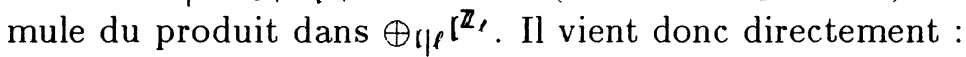

$$
r g \mathcal{N}_{K^{-}}=1+r g \mathcal{E}_{K^{-}}=r_{K}+c_{K}
$$

$\left(r_{K}:\right.$ nombre de places réelles ; $c_{K^{-}}$: nombre de places complexes de $K$ ). Plus généralement: 
ThéorÈme et DÉfinition 1.10. Pour tout corps de nombres $K$ le sousgroupe $\mathcal{N}_{K}$ du tensorisé $\mathcal{R}_{K}=\mathbb{Z}_{\ell} \otimes_{\mathbb{Z}} K^{\times}$formé des idèles principaux dont le diviseur logarithmique est trivial, est un sous-module pur de $\mathcal{R}_{K}$, produit direct $d u$ l-groupe $\mu_{K}$ des racines de l'unité dans $K$, et d'un $\mathbb{Z}_{\ell}$-module libre de dimension $r_{K}+c_{K}+\tilde{\delta}_{K}$, où $r_{K}$ est le nombre de places réelles, $c_{K}$ le nombre de places complexes et $\tilde{\delta}_{K}$ le défaut de la conjecture de Gross dans le corps $K$.

Nous disons que $\mathcal{N}_{K}$ est le groupe des unités logarithmiques de $K$.

Scolie 1.11. Avec les conventions des sections précédentes, pour chaque naturel $n$, le groupe divisible $\mathfrak{N}_{K_{n}}=\left(\mathbb{Q}_{\ell} / \mathbb{Z}_{\ell}\right) \otimes_{\mathbb{Z}_{1}} \mathcal{N}_{K_{n}}$ construit sur les unités logarithmiques du corps $K_{n}$, est un sous groupe de $\mathfrak{R}_{K_{n}}$, et un $\mathbb{Z}_{\ell^{-}}$ module divisible de codimension finie égale au nombre de places complexes du corps $K_{n}$, augmenté du défaut $\tilde{\delta}_{K_{n}}$ de la conjecture de Gross dans $K_{n}$ :

$$
\mathfrak{N}_{\kappa_{n}} \simeq\left(\mathbb{Q}_{\ell} / \mathbb{Z}_{\ell}\right)^{c_{K_{n}}+\tilde{\delta}_{K_{n}}}
$$

Lorsque celle-ci est vérifiée, les groupes $\mathfrak{N}_{K_{n}}$ et $\mathfrak{U}_{K_{n}}$ définissent le même caractère, pour tout groupe fini $\Delta_{n}$ d'automorphismes de $K_{n}$.

Démonstration : Les suites exactes précédentes montrent que le caractère du $\mathbb{Z}_{\ell}\left[\Delta_{n}\right]$-module $\mathcal{N}_{K_{n}}$ est égal à celui du module $\mathcal{E}_{K_{n}}$ des unités augmenté du caractère unité, sous réserve de la conjecture de Gross. Maintenant, le caractère de $\mathcal{E}_{K_{n}}$ est donné par le théorème de représentation de Herbrand, et celui du groupe $\mathfrak{U}_{K_{n}}$ par la théorie de Tate (cf. Scolie 1.5).

\section{c. - Application aux groupes de Brauer}

Étant donné un entier naturel $m \geq 1$, fixons $n \geq m$ pour que le corps $K_{n}$ contienne le groupe $\mu_{\ell^{m}}$ les racines $\ell^{m}$-ièmes de l'unité. L'application trilinéaire

$$
(\zeta, a, b) \mapsto[a, b]_{\zeta}
$$

qui a un élément $\zeta$ de $\bar{\mu}_{\ell^{m}}$, et à un couple $(a, b)$ d'éléments de $K_{n}^{\times}$, associe la classe dans le $\ell$-groupe de Brauer $B r\left(K_{n}\right)$ de la $K_{n}$-algèbre centrale simple construite sur deux éléments $\alpha$ et $\beta$ avec les trois relations

$$
\alpha^{\ell^{m}}=a, \quad \beta^{\ell^{m}}=b, \quad \alpha \beta=\zeta \beta \alpha
$$

définit, pour $\zeta$ fixé, un symbole sur $K_{n}$ à valeurs dans le sous-groupe de $\ell^{m}$-torsion de $\operatorname{Br}\left(K_{n}\right)$, et, en fin de compte, un morphisme non trivial du 
produit tensoriel $\bar{\mu}_{\ell^{m}} \otimes_{\mathbb{Z}} K_{2}\left(K_{n}\right)$ dans $\ell^{m} \operatorname{Br}\left(K_{n}\right)$, qui est un isomorphisme de modules galoisiens, en vertu d'un résultat de Tate (cf. [24], §5). Ce résultat est d'ailleurs extrèmement général, puisque Mercurjev et Suslin ont montré qu'il vaut en fait pour tout corps (cf. [20]).

Dans le cas des corps de nombres qui nous intéresse ici, l'isomorphisme obtenu

$$
\bar{\mu}_{\ell^{m}} \otimes_{\mathbb{Z}} K_{2}\left(K_{n}\right) \simeq_{\ell^{m}} B r\left(K_{n}\right)
$$

transporté par l'isomorphisme canonique de localisation

$$
B r\left(K_{n}\right) \simeq \underset{\bigoplus_{n} \in \operatorname{Pl}\left(K_{n}\right)}{\widetilde{P}} \operatorname{Br}\left(K_{\mathrm{p}_{n}}\right) \simeq \widetilde{\bigoplus}_{\mathfrak{p}_{n} \in \operatorname{Pl}\left(K_{n}\right)} \mathbb{Q}_{\ell} / \mathbb{Z}_{\ell}
$$

(où la somme de droite porte sur les familles $\left(b_{p_{n}}\right)_{p_{n}}$ d'éléments des $\ell$ groupes de Brauer locaux qui satisfont la formule du "produit" $\sum_{p_{n}} b_{p_{n}}=0$ dans $\mathbb{Q}_{\ell} / \mathbb{Z}_{\ell}$, lorsque $\mathfrak{p}_{n}$ décrit l'ensemble des places non complexes de $K_{n}$ ) conduit aux identifications :

$$
\begin{aligned}
{ }^{\ell^{m}} K_{2}\left(K_{n}\right)=K_{2}\left(K_{n}\right) / K_{2}\left(K_{n}\right)^{\ell^{m}} & \simeq \mu_{\ell^{m}} \otimes_{\mathbb{Z} \ell^{m}} \operatorname{Br}\left(K_{n}\right) \\
& \simeq \widetilde{\oplus}_{\mathfrak{p}_{n}} \ell^{m} \mu_{\mathfrak{p}_{n}} \simeq \widetilde{\oplus}_{\mathfrak{p}_{n} \in \Gamma \ell\left(K_{n}\right)} \mu_{\mathfrak{p}_{n}} / \mu_{\mathfrak{p}_{n}}^{\ell^{m}}
\end{aligned}
$$

Et les applications projetantes obtenues $K_{2}\left(K_{n}\right) / K_{2}\left(K_{n}\right)^{\ell^{m}} \rightarrow \mu_{\mathrm{p}_{n}} / \mu_{\mathrm{p}_{n}}^{\ell^{m}}$ ne sont rien d'autre que les morphismes induits par les symboles de Hilbert attachés aux places non complexes de $K_{n}$ (On notera que $K_{n}$ étant supposé totalement imaginaire, il n'y a pas lieu de comptabiliser de contribution réelle). C'est le résultat cité de Tate.

Considérons maintenant le groupe $\overline{K_{2}}\left(K_{n}\right)$ ou plutôt son quotient d'exposant $\ell^{m}: \ell^{m} \overline{K_{2}}\left(K_{n}\right)=\overline{K_{2}}\left(K_{n}\right) / \overline{K_{2}}\left(K_{n}\right)^{\ell^{m}}$. D'après le théorème 1.6 , c'est exactement le dual de Pontrjagin du sous-groupe de $\ell^{\boldsymbol{m}}$-torsion, disons ${ }_{\ell m}, \mathcal{X}_{K_{n}}$ du groupe de Galois $\operatorname{Gal}\left(K_{n}^{a b} / K_{\infty}\right)$ attaché à la $\ell$-extension abélienne maximale $K_{n}^{a b}$ de $K_{n}$. Maintenant, le quotient $\operatorname{Gal}\left(K_{\infty} / K_{n}\right)$ de $\operatorname{Gal}\left(K_{n}^{a b} / K_{n}\right)$ étant projectif et sans torsion, $\ell_{m} \cdot \mathcal{X}_{K_{n}}$ est aussi le sous-groupe de $\ell^{m}$-torsion du groupe $\mathcal{X}_{K_{n}}=\operatorname{Gal}\left(K_{n}^{a b} / K_{n}\right)$ qui peut être décrit par la théorie $\ell$-adique du corps de classes. D'après [13] (ch.I, section 1 ) le groupe $\mathcal{X}_{K_{n}}$ s'identifie en effet au quotient $\mathcal{J}_{K_{n}} / \mathcal{R}_{K_{n}}$ du $\ell$-adifié $\mathcal{J}_{K_{n}}=\prod_{p_{n}}^{\text {res }} \mathcal{K}_{\mathrm{p}_{n}}^{\times}$ du groupe des idèles de $K_{n}$ par le sous-groupe $\mathcal{R}_{K_{n}}=\mathbb{Z}_{\ell} \otimes_{\mathbb{Z}} K_{K_{n}}^{\times}$des idèles principaux, et les éléments de $\ell^{m}, \mathcal{X}_{K_{n}^{-}}$se trouvent représentés par les idèles $\left(\mathfrak{x}_{\left.\mathfrak{p}_{n}\right)_{\mathfrak{p}_{n}}}\right.$ dont la puissance $\ell^{m}$-ième est un élément $x$ de $\mathcal{R}_{K_{n}}$. Comme $K_{n}$ contient par construction les racines $\ell^{m}$-ièmes de l'unité, nous pouvons appliquer la théorie de Kummer qui nous dit que l'extension abélienne 
$K_{n}\left[^{e^{m}} \sqrt{x}\right]$, localement triviale partout, est globalement triviale, autrement dit que $x$ est lui-même une puissance $\ell^{m}$-ième dans $\mathcal{R}_{K_{n}}$. Écrivant alors $x=y^{\ell^{m}}$, et posant $\left(\mathfrak{y}_{\mathfrak{p}_{n}}\right)_{\mathfrak{p}_{n}}=\left(\mathfrak{x}_{\mathfrak{p}_{n}} / y\right)_{\mathfrak{p}_{n}}$, nous obtenons $\mathfrak{y}_{\mathfrak{p}_{n}}^{\ell^{m}}=1, \forall \mathfrak{p}_{n}$, ce qui nous prouve que les éléments de $\ell^{m} \mathcal{X}_{K_{n}}$ sont ceux représentés par les racines $\ell^{m}$-ièmes de l'unité dans $\mathcal{J}_{K_{n}}$ :

$$
\ell^{m} \mathcal{X}_{K_{n}} \simeq\left(\prod_{\mathfrak{p}_{n}} \ell^{m} \mu_{\mathfrak{p}_{n}}\right) /\left(\mathcal{R}_{K_{n}} \cap \prod_{\mathfrak{p}_{n}} \ell^{m} \mu_{\mathfrak{p}_{n}}\right)=\left(\prod_{\mathfrak{p}_{n}} \ell^{m} \mu_{\mathfrak{p}_{n}}\right) / \mu_{\ell^{m}} .
$$

Transportant enfin par dualité l'identité obtenue, nous obtenons l'analogue exact du théorème de Tate :

$$
\mathfrak{e}^{m} \overline{K_{2}}\left(K_{n}\right) \simeq \bigoplus_{\mathfrak{p}_{n}} \bar{\mu}_{\mathfrak{p}_{n}} / \bar{\mu}_{\mathfrak{p}_{n}}^{\ell^{m}}
$$

et, ici encore, les applications projetantes sont données par leś symboles de Hilbert loçaux. Ainsi :

ThÉorème 1.12. Pour tout $m \geq 1$ et.chaque $n \geq m$, les symboles de Hilbert attachés aux places non complexes $\mathfrak{p}_{n}$ du corps $K_{n}$ induisent les isomorphismes:

$$
K_{2}\left(K_{n}\right) / K_{2}\left(K_{n}\right)^{\ell^{m}} \simeq \widetilde{\oplus}_{\mathfrak{p}_{n}} \quad \mu_{\mathfrak{p}_{n}} / \mu_{\mathfrak{p}_{n}}^{\ell^{m}}
$$

et

$$
\overline{K_{2}}\left(K_{n}\right) / \overline{K_{2}}\left(K_{n}\right)^{\ell^{m}} \simeq \widetilde{\oplus}_{\mathfrak{p}_{n}} \quad \bar{\mu}_{\mathfrak{p}_{n}} / \bar{\mu}_{\mathfrak{p}_{n}}^{\ell^{m}}
$$

où le membre de droite est la somme directe des quotients d'exposants $\ell^{m}$ des $\ell$-groupes locaux de racines de I'unité, restreinte aux familles qui vérifient la formule du produit.

Dans les deux cas, on obtient ainsi un isomorphisme faisant intervenir le $\ell$-groupe de Brauer:

$$
\bar{\mu}_{\ell^{m}} \otimes_{\mathbb{Z}} K_{2}\left(K_{n}\right) \simeq_{\ell^{m}} \operatorname{Br}\left(K_{n}\right) \simeq \mu_{\ell^{m}} \otimes_{\mathbb{Z}} \overline{K_{2}}\left(K_{n}\right) .
$$

\section{d. - Restriction au noyau modéré (ou régulier) $\mathfrak{M}$}

Le radical universel $\mathfrak{R}$ étant très gros, il peut être commode de le remplacer par un sous-groupe canonique plus petit, le radical modéré (ou encore régulier, la distinction étant sans objet en l'absence de places réelles) $\mathfrak{M}$, défini comme suit : 
DÉfInITION 1.13. Pour tout corps de nombres $K$, I'application naturelle $x \mapsto(x)^{\prime}$ du groupe multiplicatif $K^{\times}$dans le groupe $I_{K}^{\prime}$ des idéaux inversibles du localisé $O_{K}^{\prime}=O_{K}[1 / \ell]$ en dehors de $\ell$ de l'anneau des entiers de $K$, induit une surjection du radical universel $\mathfrak{R}_{K}=\mathbb{Q}_{\ell} / \mathbb{Z}_{\ell} \otimes_{\mathbf{Z}} K^{\times}$sur le produit tensoriel $\mathbb{Q}_{\mathfrak{l}} / \mathbb{Z}_{\ell} \otimes_{\mathbb{Z}} I_{K}^{\prime}$. Son noyau

$$
\mathfrak{M}_{K}=\left\{\ell^{-k} \otimes x \in \mathfrak{R}_{K^{-}} \mid \quad(x)^{\prime} \in I_{K}^{\prime f^{k}}\right\}
$$

est appelé le sous-groupe modéré du radical $\mathfrak{R}_{K}$.

Notons d'abord que dans une tour cyclotomique $K_{\infty} / K$, les sous-groupes modérés $\mathfrak{M}_{K_{n}}$, attachés aux divers étages finis, satisfont les mêmes propriétés galoisiennes que les radicaux $\mathfrak{R}_{K_{n}}$. En effet, en l'absence de ramification en dehors de $\ell$, le fait d'être une puissance $\ell^{k}$-ième de $\ell$-idéal, pour un élément de $K_{n}^{\times}$, se lit indifférement dans $K_{n}$ ou dans $K_{\infty}$. Et la proposition 1.2 vaut donc aussi pour les sous-groupes modérés :

Proposition 1.14. Dans une tour cyclotomique, les radicaux modérés vérifient la théorie de Galois ; ce qui s'écrit :

$$
\mathfrak{M}_{\kappa_{n}}=\mathfrak{M}_{\kappa_{\infty}^{n}}^{\Gamma_{n}^{n}}, \quad \forall n \in \mathbb{N}
$$

Cela étant, tout comme leurs homologues $\mathfrak{R}_{\kappa_{n}^{-}}$les sous groupes modérés $\mathfrak{M}_{K_{n}}$ sont susceptibles d'une triple interprétation : symbolique, kummérienne, ou en termes de valeurs absolues :

- Du point de vue de la $K$-théorie, les radicaux $\mathfrak{M}_{K_{n}}$ sont les noyaux dans $\mathfrak{R}_{K_{n}}$ des $\ell$-symboles réguliers (ou modérés) à valeurs dans les $\ell$-sousgroupes de Sylow $\mu_{\mathfrak{p}_{n}}$ des groupes locaux de racines de l'unité attachés aux complétions non complexes $K_{\mathfrak{p}_{n}}$ de $K_{n}$, lesquels sont donnés par la formule bien connue :

$$
(a, b)_{\mathfrak{p}_{n}}=<(-1)^{v_{\mathfrak{p}_{n}}(a) v_{\mathfrak{p}_{n}}(b)} \frac{a^{v_{\mathfrak{p}_{n}}(b)}}{b^{v_{p_{n}}(a)}}>_{\mathfrak{p}_{n}}
$$

où $\langle.\rangle_{\mathfrak{p}_{n}}$ désigne ici la projection naturelle de $K_{\mathfrak{p}_{n}}^{\times}$sur $\mu_{\mathfrak{p}_{n}}$. Il vient donc en haut de la tour :

$$
\mathrm{T}, \otimes \mathbb{Z}, \mathfrak{M}_{K_{\infty}}=\left\{\zeta \otimes x \in \mu_{\rho} \otimes K_{\infty}^{\times} \mid \quad(\zeta, x)_{\mathfrak{p}_{\infty}}=1, \forall \mathfrak{p}_{\infty} \in \operatorname{Pl}\left(K_{\infty}\right)\right\} .
$$

Et la descente galoisienne peut s'effectuer comme suit : Partant de la suite exacte courte tordue

$$
1 \longrightarrow \mathbf{T}_{\boldsymbol{l}} \otimes_{\mathbb{Z}_{1}} \mathfrak{M}_{K_{\infty}} \longrightarrow \mu_{\rho \infty} \otimes_{\mathbb{Z}} K_{\infty}^{\times} \longrightarrow \mu_{\ell \infty} \otimes_{\mathbb{Z}} I_{\kappa_{\infty}^{\prime}}^{\prime} \longrightarrow 1,
$$


qui définit $\mathfrak{M}_{K_{\infty}}$, et prenant les points fixes par $\Gamma_{n}$, nous obtenons le diagramme commutatif

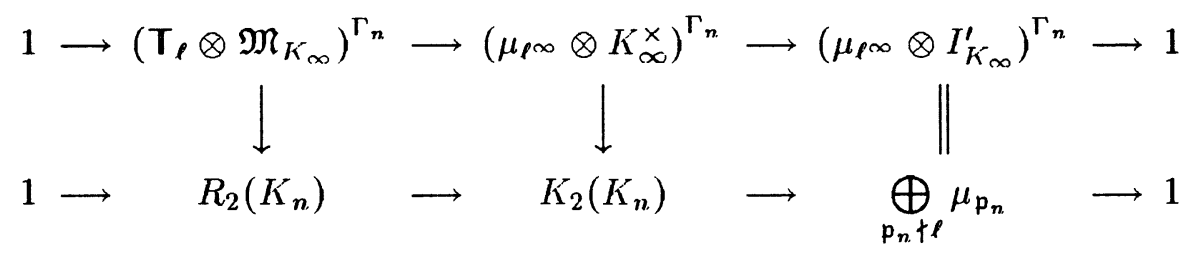

Dans la première suite, le groupe de cohomologie $H^{1}\left(\Gamma_{n}, \mathbf{T}_{\boldsymbol{l}} \otimes \mathbf{Z}_{\ell} \mathfrak{M}_{\kappa_{\infty}}\right)$ est nul par un résultat de Tate (cf.[25]), et la suite exacte du bas est induite par les symboles réguliers.

- Du point de vue kummérien, la condition de puissance $\ell^{k}$-ième de $\ell$ idéal, pour un élément $\ell^{-k} \otimes x$ de $\mathfrak{N}_{\kappa_{\infty}}$ traduit la non ramification en dehors de $\ell$ de l'extension cyclique $K_{\infty}\left[l^{\ell^{k}} \sqrt{x}\right] / K_{\infty}$. Le groupe $\overline{\mathbf{T}}_{\ell} \otimes_{\mathbf{Z}}, \mathfrak{M}_{K_{\infty}}$ est donc le radical kummérien associé à la $\ell$-extension abélienne $\ell$-ramifiée maximale $M_{\infty}$ du corps $K_{\infty}$, c'est à dire le dual de Pontrjagin du groupe de Galois $\operatorname{Gal}\left(M_{\infty} / K_{\infty}\right)$. En particulier, son sous-groupe $\left(\overline{\mathbf{T}}_{\ell} \otimes \mathbb{Z}, \mathfrak{M}_{K_{\infty}}\right)^{\Gamma_{n}}$ est lui le dual de Pontrjagin du quotient des genres $\operatorname{Gal}\left(M_{n} / K_{\infty}\right)=\Gamma_{n}$ $\left(\operatorname{Gal}\left(M_{\infty} / K_{\infty}\right)\right.$ de $\operatorname{Gal}\left(M_{\infty} / K_{\infty}\right)$ associé à $\Gamma_{n}$, c'est à dire du groupe de Galois sur $K_{\infty}$ attaché à la sous-extension maximale $M_{n}$ de $M_{\infty}$ qui est abélienne sur $K_{n}$, autrement dit à la $\ell$-extension abélienne $\ell$-ramifiée maximale $M_{n}$ de $K_{n}$. L'ensemble de cette discussion peut donc se résumer comme suit :

ThÉorème et définition 1.15. Pour chaque entier naturel $n$, le noyau $R_{2}\left(K_{n}\right)$ des symboles réguliers dans le $\ell$-groupe $K_{2}\left(K_{n}\right)$ est le noyau modéré $\left(\mathbf{T}_{\ell} \otimes \mathbb{z}, \mathfrak{M}_{K_{\infty}}\right)^{\Gamma_{n}}$ du groupe $\left(\mathbf{T}_{\ell} \otimes_{\mathbb{Z}}, \mathfrak{R}_{K_{\infty}}\right)^{\Gamma_{n}}=\left(\mu_{\ell \infty} \otimes_{\mathbb{Z}} K_{\infty}^{\times}\right)^{\Gamma_{n}} ;$ ce qui se traduit par l'exactitude de la suite courte canonique :

$$
1 \longrightarrow\left(\mathrm{T}_{\ell} \otimes \mathbb{Z}, \mathfrak{R}_{K_{\infty}}\right)_{\mathrm{div}}^{\Gamma_{n}} \longrightarrow\left(\mathrm{T}_{\ell} \otimes \mathbb{Z}_{\ell} \mathfrak{M}_{K_{\infty}}\right)^{\Gamma_{n}} \longrightarrow R_{2}\left(K_{n}\right) \longrightarrow 1
$$

Nous notons $\bar{R}_{2}\left(K_{n}\right)$ le $\ell$-groupe de torsion défini par l'exactitude de la suite courte :

$$
1 \longrightarrow\left(\overline{\mathbf{T}}_{\ell} \otimes \mathbb{Z}_{\ell}, \mathfrak{R}_{K_{\infty}}\right)_{\mathrm{div}}^{\Gamma_{n}} \longrightarrow\left(\overline{\mathbf{T}}_{\ell} \otimes \mathbb{Z}_{\ell} \mathfrak{M}_{K_{\infty}}\right)^{\Gamma_{n}} \longrightarrow \bar{R}_{2}\left(K_{n}\right) \longrightarrow 1 .
$$

Le groupe $\bar{R}_{2}\left(K_{n}\right)$ est un $\ell$-groupe fini, qui s'identifie au dual de Pontrjagin du sous-groupe de torsion $\mathcal{T}_{K_{n}}$ du groupe de Galois de la $\ell$-extension abélienne $\ell$-ramifiée maximale $M_{n}$ de $K_{n}$. 
En particulier, les groupes $R_{2}\left(K_{n}\right)$ et $\bar{R}_{2}\left(K_{n}\right)$ sont tous deux finis.

La finitude du noyau régulier $R_{2}\left(K_{n}\right)$ résulte du théorème de Garland (cf., par exemple, [2]) ; celle du groupe $\bar{R}_{2}\left(K_{n}\right)$ de la théorie du corps de classes. Dans les deux cas, on voit ainsi que le groupe tordu $\left(T_{\ell} \otimes_{\mathbb{Z}}\right.$, $\left.\mathfrak{M}_{K_{\infty}}\right)^{\Gamma_{n}}$ (respectivement $\left(\overline{\mathbb{T}}_{\ell} \otimes_{\mathbb{Z}_{\ell}}, \mathfrak{M}_{K_{\infty}}\right)^{\Gamma_{n}}$ ) est un $\mathbb{Z}_{\ell}$-module de cotype fini. Pour vérifier qu'il en va de même pour le radical $\mathfrak{M}_{\kappa_{n}}$, on peut faire appel à la suite exacte canonique

$$
1 \longrightarrow \mathfrak{E}_{K_{n}}^{\prime}=\left(\mathbb{Q}_{\ell} / \mathbb{Z}_{\ell}\right) \otimes_{\mathbb{Z}} E_{K_{n}}^{\prime} \longrightarrow \mathfrak{M}_{K_{n}} \longrightarrow C \ell_{K_{n}}^{\prime} \longrightarrow 1 .
$$

obtenue en associant à l'élément $\ell^{-k} \otimes x$ de $\mathfrak{M}_{K_{n}}$ la classe du $\ell$-idéal $\ell^{\ell^{k}} \sqrt{(x)^{\prime}}$ dans le $\ell$-groupe des $\ell$-classes d'idéaux du corps $K_{n}$, qui montre que le sous-

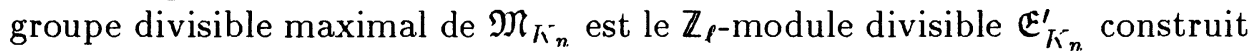
sur les $\ell$-unités de $K_{n}$. Mais ici l'analogie est imparfaite en général, parce que la codimension $c_{K_{n}^{-}}-1+s_{K_{n}}$ de $\mathfrak{E}_{K_{n}}^{\prime}$ dépasse $c_{K_{n}^{-}}$du nombre $s_{K_{n}}$ de places de $K_{n}$ au dessus de $\ell$ diminué de 1 . Enfin, il est bon de noter que les groupes $\mathfrak{E}_{K_{n}}^{\prime}$ ne vérifient pas la théorie de Galois (cf. [11], §5.4 et 6.3) :

Proposition 1.16. Dans une tour cyclotomique, les groupes divisibles $\mathfrak{E}_{K_{n}}^{\prime}=\left(\mathbb{Q}_{\ell} / \mathbb{Z}_{\ell}\right) \otimes_{\mathbb{Z}} E_{K_{n}}^{\prime}$ construits sur les $\ell$-unités ne satisfont pas en général la théorie de Galois, puisqu'on a

$$
\mathfrak{E}_{K_{\infty}}^{\prime \Gamma_{n}} / \mathfrak{E}_{K_{n}}^{\prime} \simeq \operatorname{Cap}_{K_{n}}^{\prime}
$$

où $C a p_{K_{n}}^{\prime}$ désigne le sous-groupe du groupe des $\ell$-classes $C \ell_{K_{n}}^{\prime}$ qui capitule dans $C \ell_{K_{\infty}}^{n}$.

Preuve : Cela résulte immédiatement de la suite exacte du serpent appliquée au diagramme:

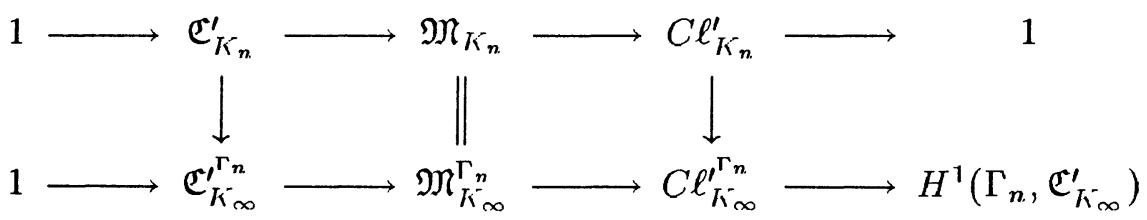

Pour obtenir une analogie plus complète dans le cas des valeurs absolues, il est ainsi nécessaire de remplacer le sous-groupe modéré par un autre, plus fin, que nous allons maintenant définir. 


\section{2. - LE RADICAL HILBERTIEN $\mathfrak{H}$}

DÉfinition 2.1. Pour chaque naturel $n$, nous appelons radical hilbertien attaché au corps $K_{n}$, et nous notons $\mathfrak{H}_{K_{n}}$ le sous-groupe du radical universel $\mathfrak{R}_{K_{n}}=\left(\boldsymbol{Q}_{\ell} / \mathbb{Z}_{\ell}\right) \otimes_{\mathbb{Z}} K_{n}^{\times}$qui est engendré par les normes cyclotomiques, i.e.

$$
\mathfrak{H}_{K_{n}}=\left\{\ell^{-k} \otimes x_{n} \in \mathfrak{R}_{K_{n}} \mid \quad \forall m \geq n \quad \exists x_{m} \in K_{m}^{\times}, x_{n} \in N_{m / n}\left(x_{m}\right) K_{n}^{\times \ell^{k}}\right\}
$$

(I'opérateur $N_{m \cdot n}$ désignant la norme arithmétique dans $K_{m} / K_{n}$ ).

La réunion $\mathfrak{H}_{\kappa_{\infty}}=\lim _{n} \mathfrak{H}_{K_{n}}$ est, par définition le radical hilbertien attaché au corps surcirculaire $K_{\infty}$.

Nous verrons un peu plus loin (cf. scolie 2.10) que les groupes $\mathfrak{H}_{\kappa_{n}}$ vérifient la théorie de Galois, tout comme les radicaux universels $\mathfrak{R}_{K_{n}}$ ou modérés $\mathfrak{M}_{K_{n}}$.

\section{a. - Interprétation symbolique : les groupes $H_{2}\left(K_{n}\right)$.}

Pour chaque place non complexe $\mathfrak{p}_{n}$ du corps $K_{n}$, désignons par $\left(\frac{1}{p_{n}}\right)$ le symbole de Hilbert associé à $\mathfrak{p}_{n}$ par le corps de classes local, ou plutôt la $\ell$-partie de ce symbole, qui prend ses valeurs dans le $\ell$-sous-groupe de Sylow $\mu_{\mathfrak{p}_{n}}$ du groupe des racines de l'unité du complété $K_{\mathfrak{p}_{n}}$ de $K_{n}$ à la place $\mathfrak{p}_{n}$. Par la propriété universelle du groupe $K_{2}\left(K_{n}\right)$ le symbole obtenu induit un morphisme surjectif du groupe $K_{2}\left(K_{n}\right)$ sur $\mu_{\mathrm{p}_{n}}$, et le théorème de Moore sur le $K_{2}$ des corps de nombres (cf. [24], §2) affirme même que la famille des symboles ainsi obtenus $\left(\left(\frac{1}{\mathfrak{p}_{n}}\right)\right)_{\mathbf{p}_{n} \in P l\left(\kappa_{n}\right)}$ induit un morphisme surjectif de $K_{2}\left(K_{n}\right)$ sur le sous-groupe $\widetilde{\oplus}_{\mathrm{p}_{n}} \mu_{\mathrm{p}_{n}}$ de la somme directe des $\mu_{\mathrm{p}_{n}}$ formé des familles $\left(\zeta_{\mathfrak{p}_{n}}\right)_{\mathfrak{p}_{n}}$ qui vérifient la forme du produit $\prod_{\mathfrak{p}_{n}} \zeta_{\mathfrak{p}_{n}}^{g_{\mathfrak{p}_{n}}}=1$ (où $g_{\mathfrak{p}_{n}}$ est l'indice de décomposition de la place $\mathfrak{p}_{n}$ dans l'extension $K_{\infty} / K_{n}$ ).

Par passage à la limite, nous obtenons ainsi un morphisme surjectif $\mathrm{du}$ $\ell$-groupe $K_{2}\left(K_{\infty}\right)=\underline{\lim } K_{2}\left(K_{n}\right)$ sur le sous-groupe $\tilde{\oplus}_{\mathrm{p}_{\infty}} \mu_{\mathrm{p}_{\infty}}$ de la somme directe des $\ell$-groupes $\overrightarrow{d e}$ racines de l'unité des complétés non complexes de $K_{\infty}$, formé des familles $\left(\zeta_{p_{\infty}}\right)_{p_{\infty}}$ qui satisfont la formule du produit

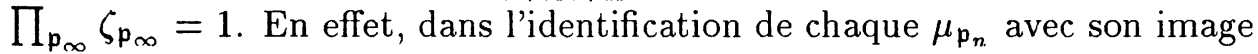
diagonale dans la somme directe $\oplus_{\mathfrak{p}_{\infty} \mid \mathfrak{p}_{n}} \mu_{\mathfrak{p}_{\infty}}$, chaque $\zeta_{\mathfrak{p}_{n}}$ est bien compté $g_{p_{n}}$ fois dans $\left.K_{\infty}\right)$.

Une autre façon d'exprimer ce résultat consiste à dire que les symboles de Hilbert $\left(\frac{1}{p_{\infty}}\right)$ attachés aux places non complexes du corps $K_{\infty}$ définissent un morphisme surjectif du groupe $\mathbb{T}_{\ell} \otimes_{\mathbb{Z}}, \mathfrak{R}_{K_{\infty}} \simeq \mu_{\ell \infty} \otimes_{\mathbb{Z}} K_{\infty}^{\times}$sur la somme restreinte $\widetilde{\oplus}_{\mathfrak{p}_{\infty}} \mu_{\mathfrak{p}_{\infty}}$. Plus précisément : 
Lemme 2.2. Le noyau de l'épimorphisme du groupe $\mathbf{T}_{\boldsymbol{\ell}} \otimes_{\mathbb{Z}}, \mathfrak{R}_{K_{\infty}}$ sur la somme restreinte $\widetilde{\oplus}_{\mathfrak{p}_{\infty}} \mu_{\mathfrak{p}_{\infty}}$, qui est donné par les symboles de Hilbert $\left(\frac{\dot{1}}{\mathfrak{p}_{\infty}}\right)$ est le sous-groupe $\mathbf{T}_{\boldsymbol{l}} \otimes_{\mathbb{Z}}, \mathfrak{H}_{K_{\infty}}$ construit sur le radical hilbertien.

Démonstration : Le noyau de l'épimorphisme ainsi construit est constitué des éléments $\zeta \otimes x$ de $\mu_{\rho \infty} \otimes_{\mathbb{Z}} K_{\infty}^{\times}$qui vérifient les conditions locales $\left(\frac{\zeta, x}{\mathfrak{p}_{m}}\right)=1$, pour chaque $m$ assez grand, c'est à dire, d'après la définition normique du symbole de Hilbert, pour lesquels le nombre $x$ est norme locale partout dans chacune des extensions cycliques $K_{m+k} / K_{m}$, si $k$ est la $\ell$-valuation de l'ordre de $\zeta$. Prenons donc un tel élément $\zeta \otimes x ;$ supposons par exemple $\zeta$ d'ordre $\ell^{k}$ et $x \in K_{n}^{\times}$; puis notons $m^{\prime}=m+k$, et invoquons le principe de Hasse pour écrire $x=N_{m^{\prime} / m}\left(x_{m}^{\prime}\right)$, avec $x_{m^{\prime}} \in K_{m^{\prime}}^{\times}$, dès que $m$ est assez grand. La condition $x^{\gamma_{n}-1}=1=N_{m^{\prime} / m}\left(x_{m^{\prime}}^{\gamma_{n}-1}\right)$, et le théorème 90 de Hilbert applique à l'extension cyclique $K_{m^{\prime}} / K_{m}$ nous permettent d'écrire $x_{m^{\prime}}^{\gamma_{n}-1}=y_{m^{\prime}}^{\gamma_{m}-1}$ pour un $y_{m^{\prime}}$ de $K_{m^{\prime}}^{\times}$, c'est à dire $x_{m^{\prime}}^{\gamma_{n}-1}=$ $N_{m / n}\left(y_{m^{\prime}}^{\gamma_{n}-1}\right)$, et finalement $x_{m^{\prime}}=N_{m / n}\left(y_{m^{\prime}}\right) . z_{m^{\prime}}$, avec $z_{m^{\prime}} \in K_{n}^{\times}$. Il suit $x=N_{m^{\prime} / m}\left(x_{m}^{\prime}\right)=N_{m^{\prime} / n}\left(y_{m^{\prime}}\right) . z_{m^{\prime}}^{\ell^{k}}$, i.e. $\zeta \otimes x=\zeta \otimes N_{m^{\prime} / n}\left(y_{m^{\prime}}\right)$; ce qui nous prouve que $\zeta \otimes x$ est bien dans $T_{\ell} \otimes \mathbb{Z}, \mathfrak{R}_{K_{\infty}}$.

Considérons maintenant la suite exacte courte donnée par le lemme :

$$
1 \longrightarrow T_{\ell} \otimes \mathbb{Z}_{\ell} \tilde{H}_{K_{\infty}} \longrightarrow \mathrm{T}_{\ell} \otimes_{\mathbb{Z}}, \mathfrak{R}_{K_{\infty}} \longrightarrow \tilde{\oplus}_{\mathfrak{p}_{\infty}} \mu_{\mathfrak{p}_{\infty}} \longrightarrow 1
$$

Prenant les points fixes par $\Gamma_{n}$, nous obtenons une suite exacte de cohomologie qui commence ainsi :

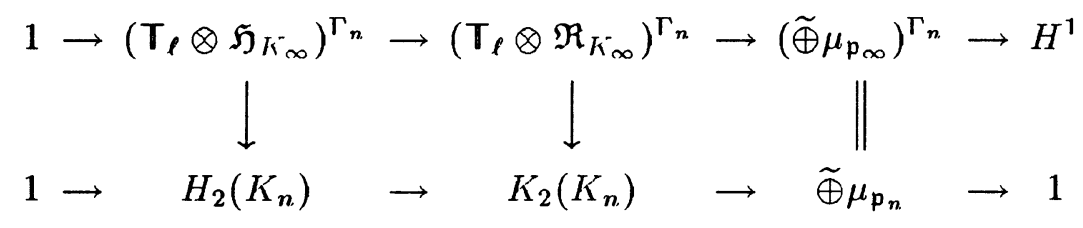

Dans celle-ci, le groupe de cohomologie

$$
H^{1}\left(\Gamma_{n}, \boldsymbol{T}_{\ell} \otimes \mathbb{Z}, \mathfrak{R}_{K_{\infty}}\right)=\left(\mu_{\rho \infty} \otimes_{\mathbb{Z}} K_{\infty}^{\times}\right) /\left(\mu_{\ell \infty} \otimes \mathbb{z} K_{\infty}^{\times}\right)^{\gamma_{n}-1}
$$

est nul, par un argument de Tate : les valeurs spectrales de l'action de $\gamma_{n}$ sur $K_{\infty}^{\times}$étant des racines de l'unité, leur produit par $\omega\left(\gamma_{n}\right)$ n'est jamais 1 , et $\gamma_{n}-1$ opère surjectivement sur le produit tensoriel $\mu_{\ell} \otimes_{\mathbb{Z}}$ $K_{\infty}^{\times}$. Ainsi, par rapprochement avec la suite exacte de Moore pour le groupe $K_{2}\left(K_{n}\right)$, écrite dessous, nous obtenons immédiatement l'identité cohomologique $H^{1}\left(\Gamma_{n}, \mathbf{T}_{\ell} \otimes_{\mathbb{Z}_{1}} \mathfrak{H}_{K_{\infty}}\right)=1$, et le théorème : 
ThÉorème 2.3. Le noyau $H_{2}\left(K_{n}\right)$ des symboles de Hilbert $\left(\frac{\boldsymbol{p}_{n}}{\mathrm{p}_{n}}\right)$ dans $K_{2}\left(K_{n}\right)$ est l'image du sous-groupe $\left(T_{\ell} \otimes \mathbb{Z}_{1}, \mathfrak{H}_{K_{\infty}}\right)^{\Gamma_{n}}$ du groupe $\left(T_{\ell} \otimes \mathbb{Z}\right.$, $\left.\mathfrak{R}_{K_{\infty}^{\prime}}\right)^{\Gamma_{n}}$, ce qui s'écrit :

$$
1 \longrightarrow\left(\mathrm{T}_{\ell} \otimes \mathbb{Z}, \mathfrak{R}_{\kappa_{\infty}}\right)_{\mathrm{div}}^{\Gamma_{n}} \longrightarrow\left(\mathrm{T}_{\ell} \otimes_{\mathbb{Z}}, \mathfrak{H}_{K_{\infty}}\right)^{\Gamma_{n}} \longrightarrow H_{2}\left(K_{n}\right) \longrightarrow 1
$$

En particulier, le groupe $\left(\mathbf{T}_{\boldsymbol{l}} \otimes_{\mathbb{Z}}, \mathfrak{H}_{K_{\infty}}\right)^{\Gamma_{n}}$ est exactement le sous-groupe des éléments de hauteur infinie dans $\left(\mathbf{T}_{\ell} \otimes_{\mathbb{Z}}, \mathfrak{R}_{K_{\infty}^{-}}\right)^{\Gamma_{n}}$.

Preuve : En effet, d'après Tate (cf. [24] $\$ 5$ ), le cas spécial étant exclu, le groupe $H_{2}\left(K_{n}\right)$ est exactement le sous-groupe des éléments de hauteur infinie dans $K_{2}\left(K_{n}\right)$. Comme tout élément divisible est trivialement de hauteur infinie, cette caractérisation du noyau hilbertien se relève dans $\left(T_{\ell} \otimes \mathbb{Z}_{,} \mathfrak{R}_{K_{\infty}}\right)^{\Gamma_{n}}$, ce qui donne le résultat annoncé.

\section{b. - Interprétation kummérienne : les groupes $\overline{H_{2}}\left(K_{n}\right)$.}

L'introduction du noyau hilbertien $\mathfrak{H}_{K}$ (sous une forme sensiblement moins générale) est due à $\mathrm{F}$. Bertrandias et $\mathrm{J}$.-J. Payan, qui avaient en vue l'étude d'une condition suffisante de la conjecture de Leopoldt, liée à un problème de plongement (cf.[3],§7). D'après Artin-Tate, en effet (cf. [1], $10 \S 3$, th. 6), une condition nécessaire et suffisante pour qu'une extention cyclique $L_{m}=K_{m}\left[e^{\ell^{m}} \sqrt{x_{m}}\right]$, définie (à composition près par $K_{\infty}$ ) par un élément $\ell^{-m} \otimes x_{m}$ de $\mathfrak{R}_{K_{m}}$ se plonge dans une $\ell$-extension cyclique de $K_{m}$ de degré arbitrairement grand est précisément que pour chaque place non complexe $\mathfrak{p}_{m}$ de $K_{m}$, la racine primitive $\zeta_{\mathfrak{p}_{m}}$ de $\mu_{\mathfrak{p}_{m}}$ soit norme dans l'extension locale $K_{\mathfrak{p}_{m}}\left[e^{e^{m}} \sqrt{x_{m}}\right] / K_{\mathfrak{p}_{m}}$, ce qui se lit sur le symbole de Hilbert $\left(\frac{\zeta_{m}, x_{m}}{\mathfrak{p}_{m}}\right)$, où $\zeta_{m}$ désigne une racine $\ell^{m}$-ième primitive de l'unité dans $K_{m}$. Plus généralement, si l'extension $L_{m}$ provient par composition avec $K_{m}$ d'une $\ell$-extension cyclique $L_{n}$ de degré $\ell^{m}$ sur un sous-corps $K_{n}$ de $K_{m}$. (i.e. si l'élément $\zeta_{m} \otimes x_{m}$ tombe dans $\left.\left(\overline{T_{\ell}} \otimes_{\mathbb{Z}_{1}} \mathfrak{R}_{K_{\infty}}\right)^{\Gamma_{n}}\right)$, le même critère appliqué à l'extension globale $L_{n} / K_{n}$ et l'équivalence donnée par le corps de classes local

$$
\left(\frac{\zeta_{\mathfrak{p}_{m}}, L_{\mathfrak{P}_{m}} / K_{\mathfrak{p}_{m}}}{\mathfrak{p}_{m}}\right)=1 \Longleftrightarrow\left(\frac{\zeta_{\mathfrak{p}_{n}}, L_{\mathfrak{p}_{n}} / K_{\mathfrak{p}_{n}}}{\mathfrak{p}_{n}}\right)=1
$$

montrent que la trivialité des symboles $\left(\frac{\zeta_{m}, x_{m}}{\mathfrak{p}_{m}}\right)$ est encore la condition pour que l'extension $L_{n}$ se plonge dans une $\ell$-extension cyclique de $K_{n}$ de degré arbitrairement grand. Autrement dit : 
Proposition 2.4. Pour chaque naturel $n$, le groupe $\left(\overline{\mathbb{T}}_{\ell} \otimes \mathbb{Z}_{\ell}, \mathfrak{H}_{K_{\infty}}\right)^{\Gamma_{n}}$ est le sous-groupe des éléments de hauteur infinie dans $\left(\overline{\mathbb{T}}_{\ell} \otimes \mathbb{Z}_{\ell} \mathfrak{R}_{K_{\infty}}\right)^{\Gamma_{n}}$.

Par analogie avec le cas des symboles, il est alors logique de poser :

ThÉORÈmE ET DÉfINITION 2.5. Par $\overline{H_{2}}\left(K_{n}\right)$ nous entendons le quotient du groupe $\left(\overline{\mathbf{T}}_{\boldsymbol{\ell}} \otimes_{\mathbb{Z}}, \mathfrak{H}_{K_{\infty}}\right)^{\Gamma_{n}}$ des éléments de hauteur infinie dans $\left(\overline{\mathbf{T}}_{\boldsymbol{\ell}} \otimes_{\mathbb{Z}_{\ell}}\right.$ $\left.\mathfrak{H}_{K_{\infty}}\right)_{\text {div }}^{\Gamma_{n}}$, par son sous-groupe divisible maximal $\left(\overline{\mathbf{T}}_{\ell} \otimes \mathbb{Z}_{\ell}, \mathfrak{R}_{K_{\infty}}\right)_{\text {div }}^{\Gamma_{n}}$, c'est à dire le $\ell$-groupe de torsion défini par l'exactitude de la suite courte :

$$
1 \longrightarrow\left(\overline{\mathbf{T}}_{\ell} \otimes \mathbb{Z}, \mathfrak{H}_{K_{\infty}}\right)_{\text {div }}^{\Gamma_{n}} \longrightarrow\left(\overline{\mathbf{T}}_{\ell} \otimes \mathbb{Z}, \mathfrak{H}_{K_{\infty}}\right)^{\Gamma_{n}} \longrightarrow \overline{H_{2}}\left(K_{n}\right) \longrightarrow 1
$$

Le groupe $\overline{H_{2}}\left(K_{n}\right)$ est l'ensemble des éléments de hauteur infinie dans $\overline{K_{2}}\left(K_{n}\right)$. C'est un $\ell$-groupe fini, qui s'identifie au dual de Pontrjagin $d u$ module de Bertrandias-Payan du corps $K_{n}$, défini comme le sous-groupe de torsion $\mathcal{T}_{K_{n}}^{\prime}=\mathcal{H}_{\kappa_{n}^{\prime}}^{\text {tor }}$ du groupe de Galois $\mathcal{H}_{\kappa_{n}^{-}}=\operatorname{Gal}\left(H_{n} / K_{n}\right)$ de la composée des $\ell$-extensions cycliques de $K_{n}$ qui se plongent dans une $\ell$-extension cyclique de $K_{n}$ de degré arbitrairement grand.

Démonstration : Nous avons vu que la théorie de Kummer identifie le groupe divisible $\left(\overline{\mathbf{T}}_{\ell} \otimes \mathbb{Z}_{\ell}, \mathfrak{R}_{\boldsymbol{K}_{\infty}}\right)_{\text {div }}^{\Gamma_{n}}$ au dual de Pontrjagin du groupe de Galois $\operatorname{Gal}\left(Z_{n} / K_{\infty}\right)$ associé à la composée $Z_{n}$ des $\mathbb{Z}_{\ell}$-extensions de $K_{n}$. Le sous-groupe des éléments de hauteur infinie dans $\left(\overline{\mathbf{T}}_{\ell} \otimes_{\mathbb{Z}}, \mathfrak{R}_{K_{\infty}^{-}}\right)^{\Gamma_{n}}$ correspond, lui, au groupe de Galois $\operatorname{Gal}\left(H_{n} / K_{\infty}\right)$ associé à la composée $H_{n}$. des $\ell$-extensions cycliques de $K_{n}$, qui sont plongeables dans une $\ell$-extension cyclique de degré arbitrairement grand sur $K_{n}$. Leur quotient $\overline{H_{2}}\left(K_{n}\right)$ est donc, comme annoncé, le dual de Pontrjagin du $\ell$-groupe de torsion $\mathcal{T}_{K_{n}}^{\prime}=\operatorname{Gal}\left(H_{n} / Z_{n}\right)$. Enfin, le fait que $\mathcal{T}_{K_{n}^{\prime}}^{\prime}$ soit fini résulte de la théorie du corps de classes : la condition de plongement qui la définit montre que l'extension $H_{n} / K_{n}$ est non ramifiée aux places modérées (i.e. étrangères à $\ell$ ), autrement dit que $H_{n}$ est contenue dans la $\ell$-extension abélienne $\ell$ ramifiée maximale $M_{n}$ de $K_{n}$, dont le groupe de Galois $\operatorname{Gal}\left(M_{n} / K_{n}\right)$ est un $\mathbb{Z}_{\mathfrak{f}}$-module neothérien, de sorte qu'il en est de même de son quotient $\mathcal{H}_{K_{n}}=\operatorname{Gal}\left(H_{n} / K_{n}\right)$.

Il est d'ailleurs possible de décrire complétement le groupe $\mathcal{H}_{K_{n}}$ en s'aidant de la théorie $\ell$-adique du corps de classes (cf. [13], ch. I.1). Par l'isomorphisme de réciprocité, en effet, le corps $H_{n}$ est associé à un sousgroupe fermé du $\ell$-adifié $\mathcal{J}_{K_{n}}=\prod_{p_{n}}^{\text {res }} \mathcal{K}_{p_{n}}^{\times}$du groupe des idèles de $K_{n}$. (défini comme le produit restreint des complétés profinis $\mathcal{K}_{\mathrm{p}_{n}}^{\times}=\varliminf_{n_{n}} K_{\mathrm{p}_{n}}^{\times} / K_{\mathrm{p}_{n}}^{\times \ell^{n}}$ des groupes multiplicatifs des complétés non complexes de $K_{n}$ ) qu'il est aisé de déterminer : la caractérisation purement locale de $H_{n}$ (via les symboles 
de Hilbert) montre que $H_{n}$ est exactement la composée des $\ell$-extensions cycliques de $K_{n}$ qui sont localement plongeables dans une $\ell$-extension cyclique de degré arbitrairement grand, c'est à dire, en fin de compte localement plongeables dans une $\mathbb{Z}_{\ell}$-extension. Du point de vue du corps de classes local, l'obstruction commune à ces plongements est, en effet, le sous-groupe de torsion $\mu_{\mathfrak{p}_{n}}$ du $\mathbb{Z}_{\ell}$-module $\mathcal{K}_{\mathrm{p}_{n}}^{\times}$. Il en résulte que le groupe de classes d'idèles qui fixe $H_{n}$ est celui engendré par les $\mu_{p_{n}}$, autrement dit que nous avons canoniquement :

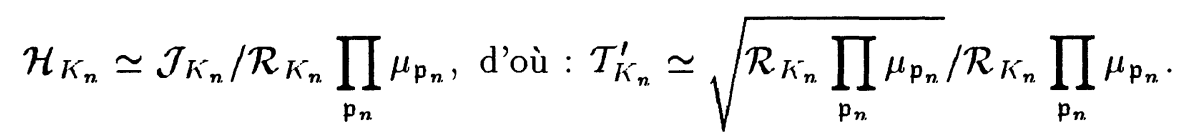

Pour réinterpréter en termes kummériens ce dernier isomorphisme, il est commode de poser :

DÉfINITION 2.6. Nous appelons sous-groupe infinitésimal du radical universel $\mathfrak{R}_{K_{n}}=\left(\mathbb{Q}_{\ell} / \mathbb{Z}_{\ell}\right) \otimes \mathbb{Z}, \mathcal{R}_{K_{n}}$, et nous notons $\widetilde{\mathfrak{C}}_{K_{n}}$ le noyau de I'application naturelle de $\mathfrak{R}_{K_{n}}$ dans le tensorisé $\mathfrak{J}_{K_{n}}=\left(\mathbb{Q}_{\ell} / \mathbb{Z}_{\ell}\right) \otimes_{\mathbb{Z}}, \mathcal{J}_{K_{n}}$ du groupe des idèles de $K_{n}$.

Par la théorie de Kummer, les éléments de $\widetilde{\mathfrak{C}}_{K_{n}}$ annulés par $\ell^{n}$ sont associés à la $\ell$-extension abélienne maximale de $K_{n}$ qui est d'exposant $\ell^{n}$ et complètement décomposée partout sur $K_{\infty}$.

Preuve : La condition $\ell^{-k} \otimes\left(x_{p_{n}}\right)_{p_{n}}=1$ pour un idèle principal $x=$ $\left(x_{\mathfrak{p}_{n}}\right)_{\mathfrak{p}_{n}}$ signifie que pour chaque place non complexe $\mathfrak{p}_{n}$, de $K_{n}$, la $\mathfrak{p}_{n^{-}}$ composante de $x$ est le produit d'une racine locale de l'unité $\zeta_{p_{n}}$ et d'une puissance $\ell^{k}$-ième, disons $y_{\mathrm{p}_{n}}^{\ell^{k}}$; de sorte que l'extension abélienne $K_{\infty}\left[l^{\ell^{k}} \sqrt{x}\right]$ est bien localement triviale partout.

Cela dit, l'isomorphisme du miroir (cf. [14], th. 10) s'énonce comme suit :

THÉorème 2.7. Iscmorphisme du Miroir - Il existe un épimorphisme canonique du radical infinitésimal $\widetilde{\mathfrak{C}}_{K_{n}}$ sur le sous-groupe de torsion $\mathcal{T}_{K_{n}}^{\prime}=\mathcal{H}_{K_{n}}^{\text {tor }}$ du groupe de Galois $\mathcal{H}_{\kappa_{n}}=\operatorname{Gal}\left(H_{n} / K_{n}\right)$, qui a pour noyau le sous-module divisible maximal $\tilde{\mathfrak{C}}_{K_{n}}^{\text {div }}$ de $\tilde{\mathfrak{C}}_{K_{n}}$ :

$$
1 \longrightarrow \widetilde{\mathfrak{C}}_{K_{n}} / \widetilde{\mathfrak{C}}_{K_{n}^{-}}^{\text {div }} \longrightarrow \mathcal{H}_{n} \longrightarrow \operatorname{Gal}\left(Z_{n} / K_{n}\right) \longrightarrow 1
$$

Le sous-module divisible $\widetilde{\mathfrak{C}}_{K_{n}}^{\text {div }}$ est nul lorsque le corps $K_{n}$ satisfait la conjecture de Leopoldt, et, dans tous les cas, sa codimension est égale au défaut $\delta_{K_{n}}$ de cette conjecture. 
Démonstration : La description idélique du radical infinitésimal donnée plus haut

$$
\tilde{\mathfrak{C}}_{K_{n}}=\left\{\ell^{-k} \otimes x \in \Re_{K_{n}} \mid x \in \mathcal{J}_{K_{n}}^{\ell^{k}} \prod_{\mathfrak{p}_{n}} \mu_{\mathfrak{p}_{n}}\right\}
$$

permet de construire aisément l'épimorphisme annoncé en associant à l'élément $\ell^{-k} \otimes x$, où l'idèle principal $x=\left(x_{p_{n}}\right)_{\mathfrak{p}_{n}}$ s'écrit localement $x_{\mathfrak{p}_{n}}=$ $y_{\mathfrak{p}_{n}}^{\ell^{k}} \zeta_{\mathfrak{p}_{n}}$, la classe modulo $\mathcal{R}_{K_{n}} \prod_{\mathfrak{p}_{n}} \mu_{\mathrm{p}_{n}}$ de l'idèle $y=\left(y_{\mathfrak{p}_{n}}\right)_{\mathfrak{p}_{n}}$, qui est bien un élément de $\mathcal{T}_{K_{n}^{-}}^{\prime}$. Is: noyau de l'application obtenue est le sous-groupe -de $\mathfrak{R}_{K_{n}}$ construit sur les éléments de $\mathcal{R}_{K_{n}}$ qui sont localement partout des racines de l'unité. Si l $\epsilon$ corps $K_{n}$ vérifie la conjecture de Leopoldt, ce sont exactement les racines; globales de l'unité, et l'épimorphisme construit est un ișómorphisme. Dans tous les cas, cependant le sous-groupe $\mathcal{R}_{K_{n}}$ formé des éléments qui sont localement partout des racines de l'unité est la racine dans $\mathcal{R}_{K_{n}}$ du sous-module $\mathcal{E}_{K_{n}}^{\text {inf }}$ des unités infinitésimales (i.e. des éléments de $\mathcal{E}_{K_{n}}=\mathbb{Z}_{\ell} \otimes_{\mathbb{Z}} E_{K_{n}}$ d'image locale 1 aux places qui divisent $\ell$ ), et la codimension du groupe divisible associé est donc bien la dimension de $\mathcal{E}_{K_{n}}^{\text {inf }}$ qui mesure par convention le défaut de la conjecture de Leopoldt dans $K_{n}^{n}$.

\section{c. - Application aux unités logarithmiques.}

Considérons le $\ell$-adifié $\mathcal{J}_{\mathcal{K}_{n}^{-}}=\prod_{p_{n}}^{\text {res }} \mathcal{K}_{\mathrm{p}_{n}}^{\times}$du groupe des idèles du corps $K_{n}$ (i.e. comme expliqué plus haut, le produit restreint des complétés profinis des groupes multiplicatifs des complétés non complexes de $K_{n}$ ), et intéressons nous au sous-groupe $\mathcal{J}_{\mathcal{K}_{n}}^{*}=\prod_{p_{n}} \mathcal{K}_{\mathrm{p}_{n}}^{*}$ construit sur les noyaux respectifs des logarithnes des valeurs absolues :

$$
\mathcal{K}_{\mathrm{p}_{n}}^{*}=\left\{x_{\mathrm{p}_{n}} \in \mathcal{K}_{\mathrm{p}_{n}}^{\times}=\underset{m}{\lim } K_{\mathrm{p}_{n}}^{\times} /\left.K_{\mathrm{p}_{n}}^{\times \ell^{m}}|\quad \log | x_{\mathrm{p}_{n}}\right|_{\mathrm{p}_{n}}=0\right\} .
$$

Distinguons les deu.: cas :

- Si la place $\mathfrak{p}_{n}$ est modérée (i.e. si $\mathfrak{p}_{n}$ est une place finie étrangère à $\ell$ ), le complété profini correspondant se décompose comme produit direct

$$
\mathcal{K}_{\mathrm{p}_{n}}^{\times}=\mu_{\mathrm{p}_{n}} \cdot \pi_{\mathrm{p}_{n}}^{\mathbb{Z}_{1}}
$$

du $\ell$-groupe fini des racines de l'unité dans $K_{p_{n}}$ et du $\mathbb{Z}_{\ell}$-module libre engendré par une uniformisante. Le sous-module $\mathcal{K}_{\mathfrak{p}_{n}}^{*}$ est donc égal à $\mu_{\mathfrak{p}_{n}}$ : c'est simultanément le sous module de torsion de $\mathcal{K}_{p_{n}}^{\times}$et son sous-groupe des unités. L'extension abélienne qui lui est associée par le corps de classes local est donc la pro- $\ell$-extension abélienne non ramifiée maximale de $K_{\mathfrak{p}_{n}}$, et sa $\mathbb{Z}_{\ell}$-extension cyclotomique. 
- Si la place $\mathfrak{p}_{n}$ est sauvage, la situation est moins simple, sauf pour $K_{\mathfrak{p}_{n}}=Q_{\ell}$ : dans ce dernier cas, en effet, le complété profini du groupe

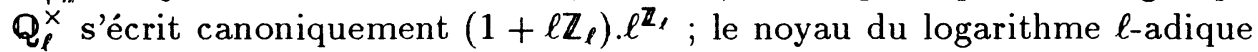
est le groupe $\ell^{Z_{1}}$; et l'extension qui lui correspond par le corps de classes local est la $\mathbb{Z}_{\ell}$-extension cyclotomique de $\mathbb{Q}_{\ell}$. En général maintenant, le sous module $\mathcal{K}_{\mathfrak{p}_{n}}^{*}$ de $\mathcal{K}_{\mathfrak{p}_{n}}^{\times}$est simplement l'image réciproque pour la norme locale $N_{K_{p_{n}}} / \mathbb{Q}_{\ell}$ de son homologue pour le corps $\mathbb{Q}_{\ell}$; et l'extension abélienne qui lui correspond est donc tout simplement la composée avec $K_{\mathfrak{p}_{n}}$ de la $\mathbb{Z}_{\ell}$.extension cyclotomique de $\mathbb{Q}_{\ell}$, c'est à dire ici encore la $\mathbb{Z}_{\ell}$-extension cyclotomique de $K_{\mathfrak{p}_{n}}$.

Dans tous les cas, il résulte du corps de classes local que $\mathcal{K}_{\mathfrak{p}_{n}}^{*}$ est le sousgroupe des normes cyclotomiques dans $\mathcal{K}_{\mathfrak{p}_{n}}^{\times}$, autrement dit que le produit

$$
\mathcal{J}_{\kappa_{n}}^{*}=\prod_{\mathfrak{p}_{n} \in \operatorname{P} \ell\left(\kappa_{n}\right)} \mathcal{K}_{\mathfrak{p}_{n}}^{*}
$$

est le sous-groupe des normes cyclotomiques locales du groupe des idèles $\mathcal{J}_{K_{n}}=\prod_{p_{n}}^{\text {res }} \mathcal{K}_{\mathbf{p}_{n}}^{\times}$.

Son intersection $\mathcal{N}_{K_{n}}=\mathcal{J}_{K_{n}}^{*} \cap \mathcal{R}_{K_{n}}$ avec le groupe des idèles principaux $\mathcal{R}_{K_{n}}=\mathbb{Z}_{\ell} \otimes_{\mathbb{Z}} K_{n}^{\times}$(intersection qui est, par définition, le sous-groupe des unités logarithmiques de $K_{n}$ ) est donc exactement, en vertu du principe de Hasse, le groupe des normes cyclotomiques globales. Ainsi :

THÉORÈmE 2.8. Les unités logarithmiques sont exactement les normes cyclotomiques.

Revenons maintenant sur la description du radical hilbertien donnée au début de la section 2. D'après la caractérisation du noyau $\mathcal{J}_{K_{n}}^{*}$ comme sous-groupe des normes cyclotomiques dans $\mathcal{J}_{K_{n}}$, la définition normique du groupe $\mathfrak{H}_{K_{n}}$ donnée plus haut

$$
\mathfrak{H}_{K_{n}}=\left\{\ell^{-k} \otimes x_{n} \in \mathfrak{R}_{K_{n}^{-}}=\left(\mathbb{Q}_{\ell} / \mathbb{Z}_{\ell}\right) \otimes_{\mathbb{Z}} K_{n}^{\times} \mid \quad x_{n} \in \bigcap_{m \geq n} N_{m / n}\left(K_{m}^{\times}\right) \cdot K_{n}^{\times \ell^{k}}\right\}
$$

s'écrit tout simplement, en termes d'idèles

$$
\mathfrak{H}_{K_{n}}=\left\{\ell^{-k} \otimes x_{n} \in \mathfrak{R}_{\kappa_{n}^{\prime}}=\left(\mathbb{Q}_{\ell} / \mathbb{Z}_{\ell}\right) \otimes_{\mathbb{Z}_{\ell}} R_{n} \mid \quad x_{n} \in \mathcal{J}_{K_{n}}^{*} \mathcal{J}_{K_{n}}^{\ell^{k}}\right\}
$$

Cela étant, il est immédiat que l'application qui à un élément $\ell^{-k} \otimes x_{n}$ du groupe $\mathfrak{R}_{K_{n}^{-}}$, sacisfaisant la condition $x_{n}=\mathfrak{x}_{n}^{*} \mathfrak{y}_{n}^{\ell^{k}} \in \mathcal{J}_{K_{n}}^{*} \mathcal{J}_{K_{n}}^{\ell^{k}}$, associe 
la classe dans $\widetilde{C \ell_{\kappa_{n}}}$ du diviseur logarithmique $h\left(\mathfrak{y}_{n}\right)$, est un morphisme surjectif du radical hilbertien $\mathfrak{H}_{K_{n}}$ sur le sous-groupe de torsion $\widetilde{C}_{K_{n}}^{\text {tor }}$ du groupe des classes logarithmiques du corps $K_{n}$, dont le noyau est constitué des éléments $\ell^{-k} \otimes x_{n}$ de $\mathfrak{R}_{K_{n}}=\left(\mathbb{Q}_{\ell} / \mathbb{Z}_{\ell}\right) \otimes \mathbb{Z}_{\ell} \mathcal{R}_{K_{n}}$ pour lesquels l'idèle principal $x_{n}$. peut être pris dans $\mathcal{J}_{K_{n}}^{*}$, c'est à dire en fin de compte dans le sous-noyau $\mathcal{N}_{K_{n}}$ des normes cyclotomiques. Autrement dit :

ThÉoRÈme 2.9. Il existe un épimorphisme canonique du radical hilbertien $\mathfrak{H}_{K_{n}}$ sur le sous groupe de torsion $\widetilde{C \ell}_{K_{n}}^{\text {tor }} d u$ groupe des classes Iógarithmiques de $K_{n}$ (en fait sur $\widetilde{C \ell}_{K_{n}}$ tout entier, sous la conjecture de Gross), qui a pour noyau le tensorisé $\mathfrak{N}_{K_{n}}=\left(\mathbb{Q}_{\ell} / \mathbb{Z}_{\ell}\right) \otimes_{\mathbb{Z}_{\ell}} \mathcal{N}_{K_{n}}$ du groupe des unités logarithmiques :

$$
1 \longrightarrow \mathfrak{N}_{K_{n}}=\left(\mathbb{Q}_{\ell} / \mathbb{Z}_{\ell}\right) \otimes \mathbb{Z}, \mathcal{N}_{K_{n}} \longrightarrow \mathfrak{H}_{K_{n}} \longrightarrow \widetilde{C}_{K_{n}}^{\text {tor }} \longrightarrow 1
$$

En particulier, le groupe $\mathfrak{N}_{K_{n}}$ est exactement le sous-module divisible maximal de $\mathfrak{H}_{\kappa_{n}^{-}}$.

Le résultat obtenu est évidemment l'analogue pour les valeurs absolues des suites exactes $(3)$ et $(\overline{3})$ ci-dessus. La suite $(\tilde{3})$ peut également être regardée comme la suite duale de celle donnée par l'isomorphisme du miroir (cf. th. 2.7).

SColie 2.10. Dans une tour cyclotomique, les radicaux hilbertiens vérifient la théorie de Galois, ce qui s'écrit :

$$
\mathfrak{H}_{K_{n}^{*}}=\mathfrak{H}_{K_{\infty}^{n}}^{\Gamma_{n}} .
$$

En revanche, les sous-groupes divisibles $\mathfrak{N}_{\kappa_{n}}$ ne la satisfont pas, puisqu'on a :

$$
\mathfrak{N}_{K_{\infty}^{\prime}}^{\Gamma_{n}} / \mathfrak{N}_{\kappa_{n}} \simeq \widetilde{\operatorname{Cap}}_{K_{n}}
$$

où $\widetilde{C a p}_{\kappa_{n}}$ désigne le sous-groupe de $\widetilde{C \ell_{K_{n}}}$ formé des classes logarithmiques qui capitulent dans $\widetilde{C}_{K_{\infty}}$.

Preuve : La description $\mathfrak{H}_{\kappa_{n}}=\left\{\ell^{-k} \otimes x_{n} \in \mathfrak{R}_{\kappa_{n}} \mid x_{n} \in \mathcal{J}_{\kappa_{n}^{*}}^{*} \mathcal{J}_{\kappa_{n}}^{\ell_{n}^{k}}\right\} \mathrm{du}$ radical hilbertien en termes idéliques montre que $\mathfrak{H}_{\kappa_{n}}$ est l'ensemble des éléments de la forme $\ell^{-k} \otimes x_{n}$, pour lesquels le diviseur logarithmique $h\left(x_{n}\right)$ associé à l'élément $x_{n}$ de $\mathcal{R}_{\kappa_{n}}=\mathbb{Z}_{\ell} \otimes_{\mathbb{Z}} K_{n}^{\times}$est une puissance $\ell^{k}$-ième dans $\mathcal{D} \ell_{K_{n}}$. Et, comme cette propriété est invariante par montée dans la tour cyclotomique, nous avons directement

$$
\mathfrak{R}_{\kappa_{n}} \cap \mathfrak{H}_{K_{m}}=\mathfrak{H}_{\kappa_{n}} \text {, pour tout } m \geq n
$$


ce qui est bien le résultat annoncé.

Cela étant, la suite exacte du serpent appliquée au diagramme

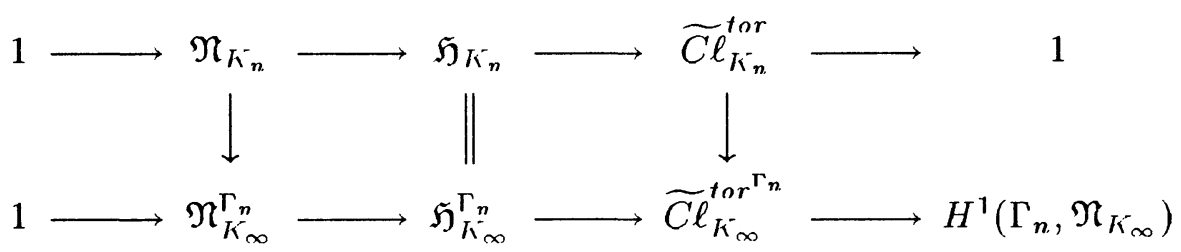

nous donne l'isomorphisme attendu

$$
\mathfrak{N}_{K_{\infty}^{n}}^{\Gamma_{n}} / \mathfrak{N}_{\kappa_{n}^{-}} \simeq \widetilde{\operatorname{Cap}}_{\kappa_{n}^{-}}
$$

puisque la capitulation pour les groupes de classes logarithmiques ne concerne en fait que les sous-groupes de torsion, de sorte que le résultat annoncé vaut indépendemment de la conjecture de Gross.

\section{d. - Isomorphismes de fausse dualité.}

Intéressons nous d'abord aux noyaux réguliers (ou modérés). Partant de la suite exacte courte de $K$-théorie donnée par les symboles modérés

$$
1 \longrightarrow R_{2}\left(K_{n}\right) \longrightarrow K_{2}\left(K_{n}\right) \stackrel{r}{\rightarrow} \bigoplus_{\mathfrak{p}_{n} \nmid \ell} \mu_{\mathfrak{p}_{n}} \longrightarrow 1
$$

prenant les puissances $\ell^{m}$-ièmes pour un $m \geq n$, puis formant la suite exacte du serpent, nous obtenons une suite exacte à six termes :

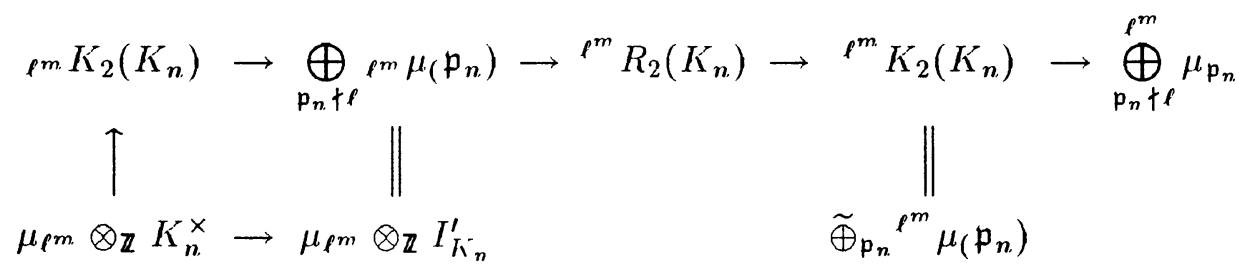
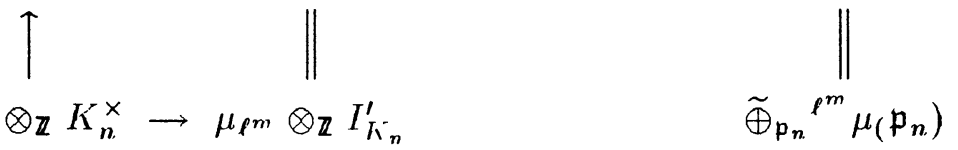

Dans celle-ci, le quotient ${ }^{f^{m}} K_{2}\left(K_{n}\right)$ s'identifie à la somme restreinte $\tilde{\oplus}_{\mathfrak{p}_{n}}$ $\ell^{m} \mu_{\mathfrak{p}_{n}}$, en vertu du théorème 1.12 ; le groupe ${ }_{\boldsymbol{f}^{m}} K_{2}\left(K_{n}\right)$ provient de $\mu_{\boldsymbol{f}^{m}} \otimes_{\mathbb{Z}}$ $K_{n}^{\times}$d'après les résultats de Tate; et la somme directe $\bigoplus_{\mathfrak{p}_{n}+\ell}^{\ell^{m}} \mu_{\mathfrak{p}_{n}}$ s'identifie 
canoniquement au produit tensoriel $\mu_{\ell^{m}} \otimes_{\mathbb{Z}} I_{K_{n}}^{\prime}$ construit sur le groupe $I_{K_{n}}^{\prime}$ des $\ell$-idéaux du corps $K_{n}$.

Rassemblant ces résultats, et remarquant que le carré de gauche est commutatif, nous en déduisons la suite exacte courte

$$
1 \longrightarrow \mu_{\ell m} \otimes_{\mathbb{Z}} C \ell_{K_{n}}^{\prime} \longrightarrow{ }^{\ell^{m}} R_{2}\left(K_{n}\right) \longrightarrow \bigoplus_{\mathfrak{l}_{n} \mid \ell}^{\ell^{m}} \mu_{\mathfrak{r}_{n}} \longrightarrow 1,
$$

qui relie le quotient d'exposant $\ell^{m}$ du groupe $R_{2}\left(K_{n}\right)$ au tensorisé $\mu_{\ell^{m}} \otimes$ $C \ell_{K_{n}}^{\prime}$ construit sur le $\ell$-groupe des classes d'idéaux du localisé en dehors de $\ell$ de l'anneau des entiers de $K_{n}$ (i.e. sur le quotient $C \ell_{K_{n}}^{\prime}$ du $\ell$-groupe des classes au sens ordinaire $C \ell_{K_{n}}$ par le sous-groupe engendré par les classes des idéaux premiers au-dessus de $\ell$ ).

Bien entendu, nous pouvons tout aussi bien écrire les mêmes suites pour les modules opposés, en partant cette fois de la suite courte (donnée encore par les symboles modérés) :

$$
1 \longrightarrow \overline{R_{2}}\left(K_{n}\right) \longrightarrow \overline{K_{2}}\left(K_{n}\right) \stackrel{\bar{r}}{\rightarrow} \bigoplus_{\mathfrak{p}_{n}+\ell} \bar{\mu}_{\mathrm{p}_{n}} \longrightarrow 1
$$

Mais ici, la surjectivité de l'application $\bar{r}$ fait problème. Transportée par dualité, elle équivaut en effet à l'injectivité de l'application naturelle du produit $\prod_{\mathfrak{p}_{n} \nmid \ell} \mu_{\mathfrak{p}_{n}}$ dans le groupe de Galois $\operatorname{Gal}\left(K_{n}^{a b} / Z_{n}\right)$. Et comme, dans l'isomorphisme $\ell$-adique du corps de classes $\mathcal{J}_{K_{n}} / \mathcal{R}_{K_{n}} \simeq \operatorname{Gal}\left(K_{n}^{a, b} / K_{n}\right)$, l'image du produit $\prod_{\mathbf{p}_{n} \nmid \ell} \mu_{p_{n}}$ n'est autre que le sous-groupe de $\operatorname{Gal}\left(K_{n}^{a b} / K_{n}\right)$ engendré topologiquement par les sous-groupes d'inertie des $\mathfrak{p}_{n}$, lequel fixe naturellement la composée $Z_{n}$ des $\mathbb{Z}_{\boldsymbol{\ell}}$-extensions de $K_{n}$, l'injectivité étudiée équivaut à celle de l'application canonique

$$
\prod_{p_{n}+\ell} \mu_{p_{n}} \longrightarrow \mathcal{J}_{K_{n}} / \mathcal{R}_{\kappa_{n}}
$$

c'est à dire, en fin de compte, à l'identité

$$
\mathcal{R}_{K_{n}} \cap \prod_{\mathfrak{p}_{n} \nmid \ell} \mu_{\mathfrak{p}_{n}}=1
$$

qui n'est rien d'autre que l'une des formulations équivalentes de la conjecture de Leopoldt : les éléments de l'intersection sont, en effet, les idèles principaux construits sur les $\ell$-unités infinitésimales (i.e. les éléments du tensorisé $\ell$-adique $\mathcal{E}_{K_{n}}^{\prime}=\mathbb{Z}_{\ell} \otimes_{\mathbb{Z}} E_{K_{n}}^{\prime}$ du groupe des $\ell$-unités, qui ont une image triviale aux places au dessus de $\ell$ ).

En résumé nous avons donc : 
Proposition 2.9. Pour chaque naturel n, les symboles réguliers (ou modérés) attachés au corps $K_{n}$ donnent naissance à la suite exacte courte de $K$-théorie :

$$
1 \longrightarrow R_{2}\left(K_{n}\right) \longrightarrow K_{2}\left(K_{n}\right) \longrightarrow \bigoplus_{p_{n}+\ell} \mu_{p_{n}} \longrightarrow 1
$$

Et la conjecture de Leopoldt dans $K_{n}$ postule qu'une suite analogue vaut pour les modules opposés :

$$
1 \longrightarrow \overline{R_{2}}\left(K_{n}\right) \longrightarrow \overline{K_{2}}\left(K_{n}\right) \longrightarrow \bigoplus_{\mathrm{p}_{n} \nmid \ell} \bar{\mu}_{\mathfrak{p}_{n}} \longrightarrow 1 .
$$

ThÉorème 2.10. (Premier isomorphisme de fausse dualité). Pour chaque naturel $n$, et chaque $m \leq n$, les symboles de Hilbert attachés aux places de $K_{n}$ au dessus de $\ell$ donnent naissance à la suite scindée :

$$
1 \longrightarrow \mu_{\ell m} \otimes_{\mathbb{Z}} C \ell_{K_{n}}^{\prime} \longrightarrow{ }^{\ell^{m}} R_{2}\left(K_{n}\right) \longrightarrow \tilde{\oplus}_{\mathfrak{r}_{n} \mid \ell}{ }^{\ell^{m}} \mu_{\mathfrak{r}_{n}} \longrightarrow 1,
$$

où $C \ell_{K_{n}}^{\prime}$ désigne le $\ell$-groupe des $\ell$-classes d'idéaux $d u$ corps $K_{n}$, et le tilde sur le signe $\oplus$ signifie que la somme est restreinte aux familles qui vérifient la formule du produit.

Lorsque le corps $K_{n}$ satisfait la conjecture de Leopoldt, la même suite vaut pour les modules opposés :

$$
1 \longrightarrow \bar{\mu}_{\ell^{m}} \otimes C \ell_{K^{-} n}^{\prime} \longrightarrow \ell^{m} \overline{R_{2}}\left(K_{n}\right) \longrightarrow \widetilde{\oplus}_{l_{n} \mid \ell^{\ell^{m}}} \bar{\mu}_{\mathfrak{l}_{n}} \longrightarrow 1,
$$

En particulier, il existe alors un isomorphisme de modules galoisiens :

$$
\mu_{\rho^{m}} \otimes_{\mathbb{Z}} \overline{R_{2}}\left(K_{n}\right) \simeq \bar{\mu}_{\ell^{m}} \otimes_{\mathbb{Z}} R_{2}\left(K_{n}\right) .
$$

Démonstration : Seul reste à vérifier que les deux suites obtenues sont effectivement scindées.

Écrivons les pour simplifier avec $m=n$, sous la forme commune

$$
1 \longrightarrow{ }^{\ell^{n}} C \ell_{\kappa_{n}}^{\prime} \longrightarrow{ }^{\ell^{n}} R_{n} \longrightarrow \widetilde{\oplus}_{l_{n} \mid \ell} \mathbb{Z} / \ell^{m} \mathbb{Z} \longrightarrow 1,
$$

En désignant par $R_{n}$ l'un quelconque des deux modules $\bar{\mu}_{\ell^{n}} \otimes \mathbb{Z} R_{2}\left(K_{n}\right)$ et $\mu_{\ell^{n}} \otimes_{\mathbb{Z}} \overline{R_{2}}\left(K_{n}\right)$. Cela étant, l'injection ${ }^{\ell^{m}} C \ell_{K_{m}}^{\prime} \hookrightarrow{ }^{\ell^{m}} R_{n}$, pour chaque $m \leq n$, montre que le groupe quotient ${ }^{\ell^{n}} C \ell_{K_{n}}^{\prime}$ est un sous-module pur de $R_{n}$, donc un facteur direct, puisque celui-ci est fini. 
Venons en maintenant aux noyaux hilbertiens : Dans le cas symbolique, le théorème de Moore sur le $K_{2}$ des corps de nombres (cf. [24]) nous donne la suite exacte courte canonique

$$
1 \longrightarrow H_{2}\left(K_{n}\right) \longrightarrow K_{2}\left(K_{n}\right) \longrightarrow \widetilde{\oplus}_{\mathfrak{p}_{n}} \mu_{\mathrm{p}_{n}} \longrightarrow 1
$$

où le terme de droite $\widetilde{\oplus}_{\mathrm{p}_{n}} \mu_{\mathrm{p}_{n}}$ représente le sous-groupe de la somme directe des $\ell$-groupes locaux de racines de l'unité attachés aux places non complexes de $K_{n}$, formé des familles $\left(\zeta_{p_{n}}\right)_{p_{n}}$ qui vérifient la formule du produit $\prod_{\mathfrak{p}_{n}} \zeta_{\mathfrak{p}_{n}}^{g_{\mathfrak{p}_{n}}}=1$, l'entier $g_{\mathfrak{p}_{n}}$ étant l'ordre du groupe de décomposition de $\mathfrak{p}_{n}$ dans l'extension procyclique $K_{\infty} / K_{n}$.

Dans le cas kummérien, les mêmes arguments que ceux développés plus haut montrent que la conjecture de Leopoldt dans $K_{n}$ affirme exactement l'existence d'une suite analogue pour les modules opposés :

$$
1 \longrightarrow \overline{H_{2}}\left(K_{n}\right) \longrightarrow \overline{K_{2}}\left(K_{n}\right) \longrightarrow \widetilde{\oplus}_{p_{n}} \bar{\mu}_{p_{n}} \longrightarrow 1
$$

Ainsi, dans l'un ou l'autre cas, prenant les puissances $\ell^{m}$-ièmes pour un $m \leq n$, et formant la suite exacte du serpent, nous obtenons une suite exacte à quatre termes de la forme :

$$
\begin{aligned}
& 1 \rightarrow \ell^{m} \dddot{H}_{2}\left(K_{n}\right) \rightarrow \ell^{m} \dddot{K}_{2}\left(K_{n}\right) \rightarrow \widetilde{\oplus}_{p_{n} \ell^{m}} \dddot{\mu}_{p_{n}} \quad \rightarrow^{\ell^{m}} \dddot{H}_{2}\left(K_{n}\right) \rightarrow 1 \\
& \dddot{\mu}_{\ell^{m}} \otimes_{\mathbb{Z}} K_{n}^{\times} \rightarrow \dddot{\mu}_{\ell^{m}} \otimes_{\mathbb{Z}} \widetilde{\mathcal{D}}_{K_{m}}
\end{aligned}
$$

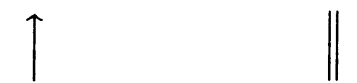

puisque le quotient d'exposant $\ell^{m} \quad \ell^{m} \dddot{K}_{2}\left(K_{n}\right)$ s'identifie à la somme restreinte $\widetilde{\oplus}_{\mathrm{p}_{n}} \ell^{m} \dddot{\mu}_{\mathrm{p}_{n}}$ d'après le théorème 1.12 .

Dans celle-ci, le terme $\ell^{m} \dddot{K}_{2}\left(K_{n}\right)$ provient du produit tensoriel $\dddot{\mu}_{\ell^{m}} \otimes_{\mathbb{Z}}$ $K_{n}^{\times}$et le terme $\widetilde{\oplus}_{\mathrm{p}_{n}, \ell^{m}} \dddot{\mu}_{\mathrm{p}_{n}}$ s'identifie canoniquement au tensorisé $\dddot{\mu}_{\ell^{m}} \otimes_{\mathbb{Z}}$

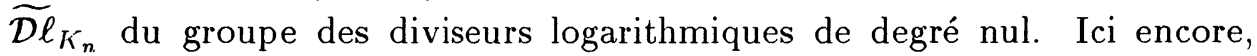
le carré au centre est commutatif, ce qui conduit en fin de compte à l'isomorphisme canonique :

$$
\ell^{m} \dddot{H}_{2}\left(K_{n}\right) \simeq \dddot{\mu}_{\ell^{m}} \otimes_{\mathbb{Z}} \widetilde{C \ell_{K_{n}}} .
$$

L'ensemble de la discussion peut donc se résumer comme suit :

Proposition 2.11. Pour chaque entier naturel $n$, les symboles de Hilbert attachés au corps $K_{n}$ donnent naissance à la suite exacte courte (de $C$. Moore) :

$$
1 \longrightarrow H_{2}\left(K_{n}\right) \longrightarrow K_{2}\left(K_{n}\right) \longrightarrow \tilde{\oplus}_{\mathfrak{p}_{n}} \mu_{\mathfrak{p}_{n}} \longrightarrow 1
$$


Et la conjecture de Leopoldt dans $K_{n}$ postule qu'une suite analogue vaut pour les modules opposés.

$$
1 \longrightarrow \overline{H_{2}}\left(K_{n}\right) \longrightarrow \overline{K_{2}}\left(K_{n}\right) \longrightarrow \tilde{\oplus}_{\mathfrak{p}_{n}} \bar{\mu}_{\mathfrak{p}_{n}} \longrightarrow 1
$$

Théorème 2.12. (Deuxième isomorphisme de dualité). - Pour chaque naturel $n$, et chaque $m \leq n$, il existe un isomorphisme de modules galoisiens du quotient d'exposant $\ell^{m}$ du noyau hilbertien de la $K$-théorie $H_{2}\left(K_{n}\right)$ associé au corps $K_{n}$ sur le tensorisé $\mu_{\mathrm{p}^{m}} \otimes_{\mathbb{Z}} \widetilde{C \ell_{K_{n}}}$ du $\ell$-groupe $\widetilde{C \ell_{K_{n}}}$ des classes logarithmiques de $K_{n}$. Et un résultat analogue vaut pour le groupe $\overline{H_{2}}\left(K_{n}\right)$ sous la conjecture de Leopoldt dans $K_{n}$, auquel cas on a :

$$
\bar{\mu}_{\ell^{m}} \otimes_{\mathbb{Z}} H_{2}\left(K_{n}\right) \simeq \ell^{m} \widetilde{C}_{K_{n}} \simeq \mu_{\ell^{m}} \otimes_{\mathbb{Z}} \overline{H_{2}}\left(K_{n}\right) .
$$

Les isomorphismes obtenus (th. 10 dans le cas régulier, th. 12 dans le cas hilbertien) permettent dans la pratique de calculer le $\ell^{m}$-rang des noyaux considérés.

\section{APPENDICE}

\section{LES CONJECTURES DE LEOPOLDT ET DE GROSS}

Nous revenons dans cet appendice sur une condition suffisante de la conjecture de Leopoldt, introduite par F. Bertrandias et J.-J. Payan [3] et déjà étudiée par R. Gillard [7] et H. Miki [21], dont nous avons dit ailleurs [12] qu'elle entraîne également la conjecture de Gross.

Nous commençons pour cela à rappeler quelques formulations équivalentes de ces conjectures faisant intervenir directement le groupe des classes logarithmiques du corps considéré.

Dans son énoncé originel, la conjecture de Leopoldt postule que le rang $\ell$-adique du groupe des unités d'un corps de nombres $K$ est égal au nombre de Dirichlet $c_{K^{-}}+r_{K}-1$, autrement dit que l'application naturelle

$$
\mathcal{E}_{K^{-}}=\mathbb{Z}_{\ell} \otimes_{\mathbb{Z}} E_{K^{-}} \longrightarrow \mathcal{U}_{K^{-}}=\prod_{\mathfrak{r} \mid \ell} U_{\mathfrak{r}}
$$

du tensorisé $\ell$-adique du groupe des unités (au sens ordinaire) de $K$ dans le groupe des unités principales du semi-localisé de $K$ au dessus de $\ell$ est un monomorphisme. Du point de vue de la théorie $\ell$-adique du corps de 
classes, les éléments de $\mathcal{E}_{K}$ étant précisément les idèles principaux $x=$ $\left(\mathfrak{x}_{\mathfrak{p}}\right)_{\mathfrak{p}} \in \mathcal{R}_{K}=\mathbb{Z}_{\mathfrak{l}} \otimes_{\mathbb{Z}} K^{\times}$qui vérifient la condition $x_{\mathfrak{p}} \in \mu_{\mathfrak{p}}$ en chaque place modérée (i.e. étrangère à $\ell$ ), la conjecture de Leopoldt revient à affirmer que les idèles principaux qui sont localement partout des racines de l'unité sont les racines globales de l'unité dans $\mathcal{R}_{K}$, et c'est sous cette forme que nous l'avons utilisée au théorème 2.7 pour montrer qu'elle équivaut en fait à la finitude du radical infinitésimal $\widetilde{C_{K}}$ attaché à $K$.

La conjecture de Gross affirme, elle, que le rang $\ell$-adique des logarithmes des valeurs absolues des $\ell$-unités d'un corps de nombres $K$ est égal au nombre $s_{K}-1$ de places de $K$ au-dessus de $\ell$ diminué de 1 , autrement dit que le sous-module du groupe $\mathcal{D} \ell_{K}$ des diviseurs logarithmiques de $K$ engendré par les diviseurs logarithmiques principaux construits sur les $\ell$-unités est un $\mathbb{Z}_{\ell}$-module libre de dimension $s_{K}-1$, ce qui revient à postuler, comme expliqué au scolie 1.9 , la finitude du groupe $\widehat{C \ell_{K}}$ des classes logarithmiques du corps $K$.

Supposons, pour simplifier, que le corps $K$ contienne les racines $2 \ell$-ièmes de l'unité. Notons $K_{\infty}=\bigcup_{n \in \mathbb{N}} K_{n}$ la $\mathbb{Z}_{\ell}$-extension cyclotomique de $K$, et $\Gamma$ le groupe procyclique $\operatorname{Gal}\left(K_{\infty} / K\right)$. Écrivons enfin

$$
\widetilde{\mathcal{C}}_{K_{\infty}}=\longleftarrow \lim \widetilde{C}_{\kappa_{n}} \quad \text { et } \quad \widetilde{\mathfrak{C}}_{K_{\infty}}=\underline{\lim } \widetilde{\mathfrak{C}}_{K_{n}}
$$

la limite projective (pour les applications normes) des $\ell$-groupes de classes logarithmiques des corps $K_{n}$ d'une part, la limite inductive (i.e. la réunion croissante) des radicaux infinitésimaux associés d'autre part. Cela posé, nous avons :

ThÉorème A.1. Dans I'isomorphisme du corps de classes, le groupe $\widetilde{\mathcal{C}}_{K_{\infty}}$ s'identifie au groupe de Galois $\operatorname{Gal}\left(K_{\infty}^{\text {loc }} / K_{\infty}\right)$ de la pro- $\ell$-extension abélienne maximale de $K_{\infty}$ qui est complètement décomposée en chacune de ses places ; et le groupe $\widetilde{\mathfrak{C}}_{K_{\infty}}$ est lui le radical kummérien $\operatorname{Rad}\left(K_{\infty}^{\text {loc }} / K_{\infty}\right)$ attaché à cette extension, de sorte que la théorie de Kummer nous donne une dualité parfaite

$$
\tilde{\mathcal{C}}_{K_{\infty}} \times \widetilde{\mathfrak{C}}_{K_{\infty}} \rightarrow \mu_{\ell \infty}
$$

à valeurs dans le groupe $\mu_{\rho \infty}$ des racines d'ordre $\ell$-primaire de l'unité.

Le groupe $\widetilde{C} \ell_{K}$ est le quotient des genres ${ }^{\Gamma} \widetilde{\mathcal{C}}_{K_{\infty}}$ attaché à $\widetilde{\mathcal{C}}_{K_{\infty}}$.

Le groupe $\widetilde{\mathfrak{C}}_{K}$ est le sous-groupe des points fixes $\widetilde{\mathfrak{C}}_{K_{\infty}}^{\Gamma}$ de $\widetilde{\mathfrak{C}}_{K_{\infty}}$.

Preuve : En termes idéliques,le groupe des classes logarithmiques $\widetilde{C} \ell_{\kappa_{n}}$ s'écrit comme le quotient $\widetilde{\mathcal{J}}_{K_{n}} / \mathcal{J}_{K_{n}^{\prime}}^{*} \mathcal{R}_{K_{n}}$ du groupe $\widetilde{\mathcal{J}}_{K_{n}}$ des idèles de $K_{n}$ 
qui vérifient la formule du produit (pour les valeurs absolues $\ell$-adiques dans $K_{n}$ ) par le sous-groupe $\mathcal{J}_{K_{n}}^{*} \mathcal{R}_{K_{n}}$ construit sur le noyau $\mathcal{J}_{K_{n}}^{*}$ des valeurs absolues. Or dans l'isomorphisme du corps de classes, $\tilde{\mathcal{J}}_{K_{n}}$ est le groupe d'idèles associé à la $\mathbb{Z}_{\ell}$-extension cyclotomique $K_{\infty}$ de $K_{n}$, et $\mathcal{J}_{K_{n}}^{*} \mathcal{R}_{K_{n}}$ celui associé à la pro- $\ell$-extension abélienne maximale $K_{n}^{\text {lor }}$ de $K_{n}$ qui est localement cyclotomique sur $K_{n}$. Par passage à la limite à partir de l'isomorphisme obtenu $\widetilde{C \ell_{K_{n}}} \simeq \operatorname{Gal}\left(K_{n}^{\text {lnc }} / K_{\infty}\right)$, nous obtenons celui annoncé $\widetilde{\mathcal{C}}_{K_{\infty}} \simeq \operatorname{Gal}\left(K_{\infty}^{\text {loc }} / K_{\infty}\right)$, puisque $K_{\infty}^{\text {loc }}$, n'est autre que la réunion des $K_{n}^{l n c}$. Bien entendu $K_{n}^{\text {lnc }}$ étant la sous-extension maximale de $K_{\infty}^{\text {lnc }}$ qui est abélienne sur $K_{n}$, nous en déduisons immédiatement l'identité des genres $\widetilde{C} \ell_{K_{n}} \simeq \widetilde{\mathcal{C}}_{K_{\infty}} / \widetilde{\mathcal{C}}_{K_{\infty} \gamma^{-1}}$, où $\gamma_{n}$ désigne un générateur topologique arbitraire du groupe procyclique $\Gamma_{n}=\operatorname{Gal}\left(K_{\infty} / K_{n}\right)$.

D'un autre côté, nous avons vu plus haut que le sous-groupe de $\ell^{n}$-torsion ${ }_{\ell^{n}} \widetilde{\mathfrak{C}}_{K_{n}}$ de $K_{n}$ est précisément le radical initial de l'extension abélienne $K_{n}^{\text {lnc }} / K_{\infty}$. Il vient donc directement $\tilde{\mathfrak{C}}_{K_{\infty}}=\operatorname{Rad}\left(K_{\infty}^{\text {lor }} / K_{\infty}\right)$, comme attendu, et inversement :

$$
\tilde{\mathfrak{C}}_{K_{\infty}}^{\Gamma_{n}}=\tilde{\mathfrak{C}}_{K_{\infty}} \cap \mathfrak{R}_{K_{n}}=\tilde{\mathfrak{C}}_{K_{n}}
$$

ce qui achève la démonstration.

Corollaire et définition A2. Pour tout corps de nombres $K$ contenant les racines $2 \ell$-ièmes de l'unité, les trois propriétés suivantes sont équivalentes :

(i) Le $\ell$-groupe des classes logarithmiques $\widetilde{C \ell}_{K}$ est trivial

(ii) Le radical infinitésimal $\tilde{\mathfrak{C}}_{K}$ est trivial

(iii) Pour qu'une $\ell$-extension abélienne de $K$ soit cyclotomique il (faut et il) suffit qu'elle le soit localement.

Lorsqu'elles sont vérifiées, nous disons que $K$ est logarithmiquement principal.

Preuve : La condition (iii) exprime la trivialité du groupe de Galois $\tilde{\mathcal{C}}_{K_{\infty}}=\operatorname{Gal}\left(K_{\infty}^{\text {lnc }} / K_{\infty}\right)$ comme du radical $\tilde{\mathfrak{C}}_{K_{\infty}}=\operatorname{Rad}\left(K_{\infty}^{l \infty} / K_{\infty}\right)$. Maintenant, la trivialité du groupe compact $\widetilde{\mathcal{C}}_{K_{\infty}}$ se lit sur le quotient des genres $\widetilde{C}_{K} ;$ celle du groupe discret $\widetilde{\mathfrak{C}}_{K_{\infty}}$ sur son sous-groupe invariant $\widetilde{\mathfrak{C}}_{K^{-}}$.

Bien entendu, le premier intérêt des corps logarithmiquement principaux est qu'ils vérifient pour des raisons purement algébriques les conjectures de Leopoldt et de Gross. Plus précisément :

Corollaire A.3. Sous les conditions équivalentes du corollaire 2, les conjectures de Leopoldt et de Gross sont satisfaites à chaque étage fini 
de la tour cyclotomique $K_{\infty} / K$, et il y a identité pour tout $n \geq 1$ entre le sous-groupe de $\ell^{n}$-torsion $\ell_{\ell^{n}} \mathfrak{H}_{K_{n}} d u$ radical hilbertien attaché au corps $K_{n}$ et chacun des trois sous-groupes suivants du radical universel $\mathfrak{R}_{K_{\infty}}=\left(\mathbb{Q}_{\ell} / \mathbb{Z}_{\ell}\right) \bigotimes_{\mathbb{Z}} K_{\infty}^{\times}$:

(i) Le sous-groupe de $\ell^{n}$-torsion $\ell^{n}\left(\overline{\mathrm{T}}_{\ell} \otimes \mathbb{Z}, \mathfrak{U}_{K_{\infty}}\right)={ }_{\ell^{n}}\left(\mathrm{~T}_{\ell} \otimes \mathbb{Z}, \mathfrak{R}_{K_{\infty}}\right)_{\text {div }}^{\Gamma_{n}}$ du noyau des symboles universels à valeurs dans $K_{2}\left(K_{n}\right)$.

(ii) Le radical initial $\ell^{n}\left(\overline{\mathrm{T}}_{\ell} \otimes \mathbb{Z}_{\ell} \mathfrak{U}_{K_{\infty}}\right)=\ell^{n}\left(\overline{\mathrm{T}}_{\ell} \otimes \mathbb{Z}_{\ell} \mathfrak{R}_{K_{\infty}}\right)_{\text {div }}^{\Gamma_{n}}$ de la composée $Z_{n}$ des $\mathbb{Z}_{\boldsymbol{p}}$-extensions du corps $K_{n}$.

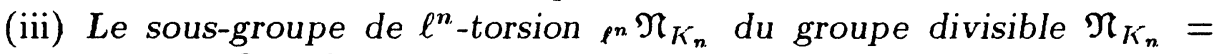
$\left(\mathbb{Q}_{\ell} / \mathbb{Z}_{\ell}\right) \bigotimes_{\mathbb{Z}} \mathcal{N}_{K_{n}}$ construit sur les normes cyclotomiques dans $K_{n}$.

Preuve : L'identité $\mathfrak{H}_{K_{n}}=\mathfrak{N}_{K_{n}}$ résulte directement du théorème 2.9. Les deux premières assertions proviennent elles du théorème 2.14 qui donne ici $H_{2}\left(K_{n}\right)=\overline{H_{2}}\left(K_{n}\right)=1$, pour tout $n$.

Remarque 1. - Par passage à la limite inductive à partir du corollaire, on obtient les identités plus faibles :

$$
\bigcup_{n \in \mathbb{N}}\left(\overline{\mathbf{T}}_{\ell} \otimes \mathbb{Z}, \Re_{K_{\infty}}\right)_{d i v}^{\Gamma_{n}}=\bigcup_{n \in \mathbb{N}}\left(\mathbb{T}_{\ell} \otimes \mathbb{Z}, \Re_{K_{\infty}}\right)_{d i v}^{\Gamma_{n}}=\mathfrak{N}_{K_{\infty}}
$$

Comme expliqué dans [14], celle de gauche est simplement la conjecture de Leopoldt écrite dans le corps $K_{\infty}$ (ce résultat est du à J. Coates (cf. [4])) ; celle de droite est la conjecture de Gross dans le même $K_{\infty}$ (ce résultat est dû̀ à $M$. Kolster (cf. [17])). En revanche, même lorsque les deux conjectures sont satisfaites dans $K_{\infty}$, l'égalité des trois radicaux du corollaire pose problème en général puisque la redescente impose une torsion de l'action galoisienne conformément aux inclusions (cf. [14], §6) :

$$
\begin{aligned}
& \ell^{n}\left(\mathbb{T}_{\ell} \otimes \mathbb{Z}, \Re_{K_{\infty}}\right)_{d i v}^{\Gamma_{n}} \subset \ell^{n}\left(T_{\ell} \otimes_{\mathbb{Z}}, \mathfrak{N}_{K_{\infty}}\right)^{\Gamma_{n}}, \\
& \ell^{n}\left(\bar{T}_{\ell} \otimes_{\mathbb{Z}}, \mathfrak{R}_{K_{\infty}}\right)_{\text {div }}^{\Gamma_{n}} \subset \ell^{n}\left(\bar{T}_{\ell} \otimes_{\mathbb{Z}}, \mathfrak{N}_{K_{\infty}}\right)^{\Gamma_{n}}
\end{aligned}
$$

et bien entendu :

$$
\ell^{n} \mathfrak{N}_{K_{n}} \subset \ell^{n} \mathfrak{N}_{K_{\infty}}^{\Gamma_{n}}
$$

Remarque 2. - En présence des racines $2 \ell$-ièmes de l'unité (en fait sous une condition plus faible) l'identité ${\widetilde{C} \ell_{K}}^{-}=1$ équivaut à la nullité $H_{2}(K)=$ $1 \mathrm{du} \ell$-groupe de Sylow du noyau hilbertien. Si l'on remplace cette condition par la condition plus forte $R_{2}(K)=1$, on tombe sur la notion de corps $\ell$ régulier (ou $\ell$-rationnel) étudiée en collaboration avec $G$. Gras dans [9] et, indépendemment par H. Movahhedi et T. Nguyen Quang Do (cf. [15]). Ceci permet de construire très facilement des familles infinies de corps non abéliens qui satisfont les conjectures de Leopoldt et de Gross. 


\section{INDEX DES NOTATIONS}

\section{Notations latines}

$K$ : un corps de nombres

$K_{\infty}$ : sa $\mathbb{Z}_{\ell}$ extension cyclotomique

$K_{n}$ : la sous-extension $K\left[\mu_{\ell^{n}}\right]$

$M_{n}$ : la $\ell$-extension abélienne $\ell$-ramifiée maximale de $K_{n}$

$H_{n}$ : la composée des extensions de $K_{n}$ localement $\mathbb{Z}_{\boldsymbol{\ell}}$-plongeables

$Z_{n}$ : la composée des $\mathbb{Z}_{\ell}$-extensions

$K_{n}^{\text {loc }}$ : la $\ell$-extension abélienne maximale localement cyclotomique

$E_{K_{n}}:$ le groupe des unités de $K_{n}$

$E_{K_{n}}^{\prime}:$ le groupe des $\ell$-unités $c_{K_{n}}, r_{K_{n}^{-}}, s_{K_{n}^{-}}$: les nombres de places complexes, réelles, ou sauvages.

\section{Notations anglaises}

$\mathcal{J}_{K_{n}}$ : le $\ell$-adifié des idèles de $K_{n}$ $\mathcal{U}_{K_{n}}$ : le sous-groupe unité de $\mathcal{J}_{K_{n}}$ $\mathcal{J}_{K_{n}^{*}}^{*}$ : le noyau des valeurs absolues $\mathcal{R}_{\kappa_{n}}$ : le sous-groupe principal $\mathcal{E}_{K_{n}^{-}}$: le groupe des unités de $\mathcal{R}_{K_{n}}$ $\mathcal{E}_{K_{n}}^{\prime}$ : le groupe des $\ell$-unités de $\mathcal{R}_{K_{n}}$ $\mathcal{N}_{K_{n}}$ : les normes cyclotomiques

\section{Notations gothiques}

$\tilde{\mathfrak{C}}_{K_{n}}$ : le radical logarithmique $\mathfrak{E}_{K_{n}}$ : le tensorisé $\left(\mathbb{Q}_{\boldsymbol{f}} / \mathbb{Z}_{\mathfrak{\ell}}\right) \otimes_{\mathbb{Z}} E_{K_{n}}$ $\mathfrak{E}_{K_{n}}^{\prime}:$ le tensorisé $\left(\mathbb{Q}_{\ell} / \mathbb{Z}_{\ell}\right) \otimes_{\mathbb{Z}} E_{K_{n}}^{\prime}$ $\mathfrak{N}_{K_{n}}$ : le tensorisé $\left(\mathbb{Q}_{\ell} / \mathbb{Z}_{\ell}\right) \otimes_{\mathbb{Z}}, \mathcal{N}_{K_{n}}^{n}$ $\mathfrak{J}_{\kappa_{n}}$ : le tensorisé $\left(\mathbb{Q}_{\ell} / \mathbb{Z}_{\ell}\right) \otimes_{\mathbb{Z}}, \mathcal{J}_{K_{n}}$

\section{Notations grecques}

$\Gamma$ : le groupe profini $\operatorname{Gal}\left(K_{\infty} / K\right)$

$\Gamma_{n}$ : le sous-groupe $\operatorname{Gal}\left(K_{\infty} / K_{n}\right)$ $\mu_{\ell^{n}}$ : le groupe des racines $\ell^{n}$-ièmes $\delta_{K_{n}}$ : le défaut de Leopoldt
$C \ell_{K_{n}}:$ le $\ell$-groupe des classes $C \ell_{K_{n}}^{\prime}$ : le $\ell$-groupe des $\ell$-classes $\widetilde{C}_{K_{n}^{\prime}}$ : les classes logarithmiques $K_{2}\left(K_{n}\right)$ : le $\ell$-groupe des symboles $\overline{K_{2}}\left(K_{n}\right)$ : le $\ell$-groupe opposé $R_{2}\left(K_{n}\right)$ : le noyau régulier dans $K_{2}$ $\mathrm{H}_{2}\left(K_{n}\right)$ : le sous-noyau hilbertien $\bar{R}_{2}\left(K_{n}\right)$ : le noyau régulier dans $\bar{K}_{2}$ $\bar{H}_{2}\left(K_{n}\right)$ : le sous-noyau hilbertien $I_{K_{n}}^{\prime}$ : le groupe des $\ell$-idéaux de $K_{n}$ $T_{\ell}$ : le module de Tate

$\overline{\mathrm{T}}_{\ell}$ : le module opposé

$\mathcal{D} \ell_{K_{n}}$ : les diviseurs logarithmiques

$\widetilde{\mathcal{D} \ell_{K_{n}}}$ : le noyau du degré dans $\mathcal{D} \ell$

$\mathcal{P} \ell_{K_{n}}$ : le sous-groupe principal

$\mathcal{K}_{\mathfrak{p}_{n}}^{\times}$: le complété $\ell$-adique de $K_{\mathfrak{p}_{n}}^{\times}$

$\mathcal{K}_{p_{n}}^{*}$ : le noyau de la valeur absolue

$\mathcal{U}_{p_{n}}$ : le sous-groupe unité de $K_{p_{n}}^{\times}$

$\mu_{p_{n}}:$ le sous-groupe fini de $K_{p_{n}}^{\times}$

$\mathfrak{R}_{K_{n}^{-}}$: le radical universel de $K_{n}$ $\mathfrak{M}_{\kappa_{n}^{-}}$: le radical modéré $\mathfrak{H}_{\kappa_{n}^{-}}$: le radical hilbertien $\mathfrak{Z}_{K_{n}}$ : le radical des $\mathbb{Z}_{\boldsymbol{\ell}}$-extensions $\mathfrak{U}_{\kappa_{n}}$ : le noyau universel

$\gamma$ : un pro-générateur de $\Gamma$ $\gamma_{n}$ : un pro-générateur de $\Gamma_{n}$ $\mu_{\rho \infty}$ : la réunion des $\mu_{\rho^{n}}$ $\widetilde{\delta}_{K_{n}}:$ le défaut de Gross 


\section{RÉFÉRENCES}

[1] E. Artin \& J. Tate, "Class field theory," Benjamin, New York-Amsterdam, 1967.

[2] H. Bass, $K_{2}$ des corps globaux, [d'après J. Tate, H. Garland...] Sém. Bourbaki, $23^{\text {ème }}$ année $(1970 / 71) n^{\circ} 394$.

[3] F. Bertrandias \& J.-J. Payan, $\Gamma$-extensions et invariants cyclotomiques, Ann. Sc. Ec. Norm. Sup. 5 (1972), 517-548.

[4] J. Coates, $\mathrm{On}_{2}$ and some classical conjectures in algebraic number theory, Ann. Math. 95 (1972), 99-116.

[5] L.J. Federer, The non-vanishing of Gross p-adic regulator Galois cohomologically, Astérisque 147-148 (1987), 71-77.

[6] L.J. Federer \& B.H. Gross (avec un appendice de W. Sinnot), Regulators and Iwasawa modules, Inv. Math. 62 (1981), 443-457.

[7] R. Gillard, Formulations de la conjecture de Leopoldt et étude d'une condition suffisante, Abh. Math. Sem. Hamburg 48 (1979), 125-138.

[8] G. Gras, Plongements kummériens dans les $\mathbb{Z}_{p}$-extensions, Compositio Math. $\mathbf{5 5}$ (1985), 383-395.

[9] G. Gras \& J.-F. Jaulent, Sur le corps de nombres réguliers, Math. Z 202 (1989), 343-365.

[10] R. Greenberg, $A$ note on $K_{2}$ and the theory of $\mathbb{Z}_{p}$-extensions, Am. J. Math. 100 (1978), 1235-1245.

[11] K. Iwasawa, On $\mathbb{Z}_{\ell}$-extensions of number fields, Ann. of Math. 98 (1973), 257-274.

[12] J.-F. Jaulent, Sur les conjectures de Leopoldt et de Gross, Astérisque 147-148 (1987), 107-120.

[13] J.-F. Jaulent, L'arithmétique des $\ell$-extensions (thèse), Pub. Math. Fac. Sci. Besançon, Théor. Nombres 1984-85 et 1985-86, fasc.1 (1986), 1-349.

[14] J.-F. Jaulent, Noyau universel et valeurs absolues, in Journées arithmétiques de Marseille Luminy, Prépublication.

[15] J.-F. Jaulent \& T. Nguyen Quang do, Corps p-rationnels, corps p-réguliers, et ramification restreinte, Sém. Théorie des Nombres Bordeaux 1987-1988, exp. $\mathrm{n}^{\circ} 10$ (1988),

[16] F. Keune, On the structure of $K_{2}$ of the ring of integers in a number field, Prépublication.

[17] M. Kolster, On idelic approach to the wild kernel, Prépublication.

[18] K. Kramer, On the Hilbert kernel of K-theory and the Gross regulator, Prépublication.

[19] K. Kramer \& A. Candiotti, On $K_{2}$ and $\mathbb{Z}_{\ell}$-extensions of number fields, Am. J. Math. 100 (1978), 177-193.

[20] A.-S. Mercurjev \& A.A. Suslin, K-cohomology of Severi-Brauer varieties and norm residue homomorphism, Izv. AN SSSR 46, NS (1982), 1011-1046.

[21] H. Miki, On the maximal abelian $\ell$-extension of a finite algebraic number field 
with given ramification, Nagoya Math. J. 70 (1978), 183-202.

[22] T. Nguyen Quang do, Sur la torsion de certains modules galoisiens p-ramifiés, Théorie des Nombres (Quebec, PQ,1987) 740-754 (1989), de Gruyter, Berlin-New York.

[23] T. Nguyen Quang do, Sur la torsion de certains modules galoisiens II, Sém. Théorie des Nombres Paris 1986-87; Progress in Math. 75 (1988), 271-298.

[24] J. Tate, Symbols in arithmetics, Actes Congrès International Math. 1 (1970), 201-211.

[25] J. Tate, Letter from Tate to Iwasawa on a relation between $K_{2}$ and Galois cohomology-Algebraic K-theory II, Lecture Notes in Math. 342 (1973).

[26] J. Tate, Relations between $K_{2}$ and Galois cohomology, Invent. Math. 36 (1976), 257-274.

[27] J. Tate, Les conjectures de Stark sur les fonctions $L$ d'Artin en $s=0$, Progress in Math. 47 (1984), Birkhäuser.

\author{
Jean-François JAULENT \\ Centre de Recherche en Mathématiques de Bordeaux \\ Université Bordeaux I \\ 351, cours de la Libération \\ F 33405 TALENCE CEDEX.
}

